\title{
Article \\ Smart Recommendations for Renting Bikes in Bike-Sharing Systems
}

\author{
Holger Billhardt, Alberto Fernández * $*$ and Sascha Ossowski \\ CETINIA, Universidad Rey Juan Carlos, 28933 Móstoles, Madrid, Spain; holger.billhardt@urjc.es (H.B.); \\ sascha.ossowski@urjc.es (S.O.) \\ * Correspondence: alberto.fernandez@urjc.es
}

Citation: Billhardt, H.; Fernández,

A.; Ossowski, S. Smart

Recommendations for Renting Bikes in Bike-Sharing Systems. Appl. Sci. 2021, 11, 9654. https://doi.org/ 10.3390/app11209654

Academic Editors: Juan-Carlos Cano and Paola Pellegrini

Received: 21 September 2021

Accepted: 14 October 2021

Published: 16 October 2021

Publisher's Note: MDPI stays neutral with regard to jurisdictional claims in published maps and institutional affiliations.

Copyright: (c) 2021 by the authors. Licensee MDPI, Basel, Switzerland. This article is an open access article distributed under the terms and conditions of the Creative Commons Attribution (CC BY) license (https:/ / creativecommons.org/licenses/by/ $4.0 /)$.

\begin{abstract}
Vehicle-sharing systems-such as bike-, car-, or motorcycle-sharing systems-have become increasingly popular in big cities in recent years. On the one hand, they provide a cheaper and environmentally friendlier means of transportation than private cars, and on the other hand, they satisfy the individual mobility demands of citizens better than traditional public transport systems. One of their advantages in this regard is their availability, e.g., the possibility of taking (or leaving) a vehicle almost anywhere in a city. This availability obviously depends on different strategic and operational management decisions and policies, such as the dimension of the fleet or the (re)distribution of vehicles. Agglutination problems-where, due to usage patterns, available vehicles are concentrated in certain areas, whereas no vehicles are available in others-are quite common in such systems, and need to be dealt with. Research has been dedicated to this problem, specifying different techniques to reduce imbalanced situations. In this paper, we present and compare strategies for recommending stations to users who wish to rent or return bikes in station-based bike-sharing systems. Our first contribution is a novel recommendation strategy based on queuing theory that recommends stations based on their utility to the user in terms of lower distance and higher probability of finding a bike or slot. Then, we go one step further, defining a strategy that recommends stations by combining the utility of a particular user with the utility of the global system, measured in terms of the improvement in the distribution of bikes and slots with respect to the expected future demand, with the aim of implicitly avoiding or alleviating balancing problems. We present several experiments to evaluate our proposal with real data from the bike sharing system BiciMAD in Madrid.
\end{abstract}

Keywords: bike sharing; agent-based coordination; smart transportation; smart mobility; multiagent systems

\section{Introduction}

Due to the growing need for reducing air pollution and, at the same time, offering mobility services of satisfactory quality to citizens, new means of urban mobility are being explored in many large cities around the world. Bike-sharing systems (BSSs) are just one of the many solutions that have been proposed and implemented in recent years. In some cities, they have grown to considerable size. For example, the system Vélib (http: / / www.velib-metropole.fr /) in Paris has 15,000 bikes, the Santander Cycles project in London (tfl.gov.uk/modes/cycling/santander-cycles) has 11,000 bikes and cities in China such as Hangzhou or Wuhan employ as many as 78,000 and 90,000 bikes, respectively [1]. Some of those systems are so called free-floating systems (e.g., BikeShare in Seattle or DB Callabike in Munich) that allow citizens to pick up and return a bike at any location within a certain area. Others are station-based-that is, they rely on a set of rental stations with fixed locations. Station-based BSSs have the advantage of providing better control to avoid unauthorized access. When electric bikes are deployed in the BSS-as, for example, in the BiciMAD system in Madrid (Spain)—stations usually serve as charging spots for the bicycles' batteries as well. 
Effectiveness and user satisfaction within station-based BSSs strongly depend on the availability of bikes (or slots for returning rented bikes) at the places where users search for them or demand them. Therefore, proper management policies need to be implemented not only for strategic decisions (e.g., regarding the positioning and dimensioning of rental stations, the selection of adequate bicycle models, etc.), but also at the operational level. In particular, BSSs are usually faced with agglomeration or unbalancing problems: available bikes are concentrated at certain areas (and at certain times of the day), whereas in other areas few or no bikes are available. The effect is that users may experience a poor quality of service, being unable to find a bike (or a return slot) at a given station or area. Solutions such as reserving bikes (or slots) at some stations palliate this problem, but proper bike balancing mechanisms are needed in order to tackle the problem at its core.

In many systems, trucks are used to address such balancing problems, by moving bicycles from (almost) full to (almost) empty stations. Truck movements are scheduled either statically (usually at night) or dynamically (during BSS operation), with the aim of finding a near-optimal match between the expected demand and the available resources. However, truck movements are expensive, and raise environmental concerns.

Today, BSSs are often conceived as cyber-physical systems [2,3]. Bikes may be endowed with several battery-powered sensors that measure important information such as position or battery level (in case of electric bicycles). Sensors at stations may capture rental attempts that were frustrated-e.g., due to mechanical failures of slot clamps-and, of course, gather key information regarding the operating state of the system, such as the numbers of available and occupied slots. This information is then transmitted to a software application at the BSS control centre, and helps operators to make timely management decisions in an online manner. Part of this information is often shared with the BSS users, who may use smartphone apps that inform them about the current state of stations in real time. Users can then use this information to make more informed decisions as to where to rent or to return a bike. However, due to the dynamic nature of the BSS environment, it often transpires that, when a user reaches a certain station, initially available bikes have already been taken by other users. Similarly, information on available parking slots may already be outdated when a user arrives to a station. Dynamic reservation schemes can be used to address such problems, but may have a negative impact on the overall capacity of a BSS.

BSSs are also often perceived from the perspective of the smart cities paradigm as part of a more sophisticated, heterogeneous, intelligent transportation solution-a system of systems that integrates different modes of transport (buses, trains, private and shared vehicles, on-demand transportation services, etc.) and enables dynamic collaborations among users, such as crowdshipping [4] or crowdsensing [5,6] scenarios. In a multimodal context $[7,8]$, real-time recommendation systems may suggest any type of appropriate means of transport (not just bikes) that fits the particular needs and preferences of a user at a particular moment. In some cases, this may alleviate the quality of service problems within a BSS due to imbalances, as mentioned above. For example, if there are no available bikes in the area of a user, the system may recommend an on-demand shared taxi service, instead of walking to another BSS station. This perspective is very appealing, and research in this direction will pave the way towards better and greener transportation services in smart cities [9,10]; however, it does not explicitly account for the agglomeration problems that reduce the quality of service at the level of the BSS. Therefore, we argue that addressing the imbalance problem in BSSs is still a relevant endeavour, as it can impact positively on the overall service level of intelligent transportation systems within a smart city.

In this work, we focus on the aforementioned problems within station-based BSSs. Our first contribution is a novel recommendation strategy based on a notion of cost that combines distance (between a user's origin/destination and a rental/return station) with (an expectation of) availability of slots or bikes. To account for the dynamic nature of the BSS environment as mentioned earlier, instead of recommending stations that have available bikes or slots at the time a search request is made, we use demand prediction to estimate 
the expected probability of finding an available bike or slot at the time the user will arrive at a recommended station. In particular, we model stations as queues, and apply techniques from queuing theory for probability estimation.

The second contribution of this work is an extension of the aforementioned recommendation strategy that also addresses balancing problems by combining individual objectives with social welfare. For this purpose, the system not only tries to recommend stations with a low cost for the user, but also uses demand predictions to estimate the impact of renting or returning a bike at a given station on society (potential future users). Here, recommendations prioritise stations (for rental or return actions) that combine a low cost for the user with an improved overall impact on the distribution of bikes and slots in the BSS (and, thus, for potential future users).

To evaluate the proposed recommendation strategies, we used a simulation tool for station-based bike-sharing systems: Bike3S (https://github.com/gia-urjc/Bike3SSimulator). This simulator performs semi-realistic simulations of the operation of a bikesharing system in a given area of interest, and allows for evaluating and testing different management decisions and strategies. In our simulations, we used real data from the BiciMAD BSS, which is operating in Madrid (Spain).

The rest of the paper is organised as follows: In Section 2 we discuss related works on bicycle-sharing systems. Section 3 outlines the general functioning of a station-based BSS, and introduces the basic notation used in the rest of the paper. Section 4 focuses on station recommendation strategies that are centred on BSS users. In particular, we sketch some simple, straightforward selection strategies, before introducing our more sophisticated proposal. Section 5 puts forward several experiments that compare the performance of the different strategies. In Section 6, we present our estimation of the impact of a rental/return action at a station on potential future users, and define our proposal for a station recommendation strategy that combines cost for users and future global impact. In Section 7, we analyse the performance of this strategy in a set of simulation experiments. Finally, Section 8 summarises our work, outlines the lessons we have learned, and points to future lines of work.

\section{Related Work}

Bike-sharing systems' management decisions can be made with different temporal horizons.

Long-term or strategic decisions are mainly related to system design and deployment (location and size of stations, number of bikes, etc.) [11-14], as well as the analysis of different exploitation policies for existing systems [15].

At an operational level, decisions are made in order to maintain a good performance of the system, which usually means maximizing the number of users that rent bikes. Thus, actions are aimed at reducing imbalances produced at stations due to heterogeneous demand for bikes and empty slots. In this regard, offline or static approaches are mid-term decisions oriented at repositioning bikes using vehicles (trucks) - typically at night, or during off-peak times-in such a way that bike distribution through stations is optimal for the expected demand. These systems estimate the best way of distributing not only the existing bikes, but also the routes taken by the trucks (see e.g., [16-18]).

A natural evolution of such systems is their online application during the actual operation of the fleet. The challenge is in combining the current state of the system with demand prediction, so as to propose short-term balancing actions. Many works propose solutions to the problem of dynamically repositioning bikes using trucks as efficiently as possible [19-22]. It has become clear that optimal solutions to the optimization problem do not scale as the size of the BSS grows, so authors use heuristics to find solutions applicable in real-world systems.

Furthermore, using trucks to rebalance a bike-sharing fleet has several disadvantages, including cost of operation, contribution to congestion and pollution, etc. Recently, several approaches have proposed crowdsourcing this task, so that users can contribute by taking 
or returning bikes at stations other than their target station. Typically, users receive some type of incentive (e.g., discounts) to compensate the extra (usually small) effort of modifying their initial choices.

This paper focuses on dynamic rebalancing systems; thus, we describe related works in this area in further detail.

Chemla et al. [19] studied the problem of obtaining the optimal policy to determine the best action that a repositioning vehicle can take in order to maximise the number of users that find a bike. Since this is an NP-hard problem, they proposed several heuristic solutions that, roughly speaking, are based on choosing the station with the most or least excess of bikes with regard to a given target state. They also employed a pricing strategy in which each station has a price that is paid when the bike is returned, regardless of the origin of the trip; users choose the destination station based on its current price and the user's walking and biking distances. This solution consists of optimizing station prices assuming certain user models.

Pfrommer et al. [23] presented a dynamic rebalancing system that combines truckbased vehicle redistribution and price incentives for users. In this approach, users either find a bike at their origin stations or leave the system. Upon arrival at their target station, users decide whether or not to accept an incentive to return the bike at a neighbouring station. This decision is taken according to their "perceived cost" of the additional distance travelled (biking and walking) and the incentives received. Dynamic prices are calculated through an optimization problem that combines the quality of the resulting state and the cost of paying out the incentives. The key component of the system quality is the analysis of each station's utility, based on an optimal "fill level" (the available bikes at a station).

Haider et al. [24] proposed a method that combines trucks and price incentives. In this approach, the authors intentionally tried to make some stations even more imbalanced (hubs), so that bike redistribution could be carried out more efficiently with shorter truck trips. The objective was to minimize the number of imbalanced stations, which the authors solved heuristically. Prices (which include incentives) were established for O-D station pairs.

Singla et al. [25] presented a mechanism that incentivizes the users in the bike repositioning process; they employed optimal pricing policies using regret minimization in online learning, and investigated the incentive compatibility of their mechanism. This approach was evaluated through simulations based on historical data from the Boston Hubway system, as well as on data collected via a survey study with MVGmeinRad in Mainz, Germany.

Reiss and Bogenberger [26] proposed a hybrid approach for a free-floating bikesharing system in which user discounts (60-100\%) are applied when the imbalance is low (less than 15\%), or trucks are used in case of high imbalances. The decision is updated once for each of five predefined time slots during the day. Imbalances are measured as the difference between the current and a desired fleet distribution. The latter is calculated for the entire day from historical data.

Wang and Hou [27] proposed a repositioning strategy that assumes that there is a number of voluntary riders during each time period. Trips (including bike repositioning by volunteers) are considered for time periods of $30 \mathrm{~min}$. The authors used integer programming to solve two optimization problems: first (1) the "ideal bike inventory" for each station and time period (with unlimited voluntary riders), and then (2) the voluntary rider flow (O-D pairs), so as to obtain an inventory as close to the "ideal" as possible.

Chung et al. [28] analysed Bike Angels-the incentive program of New York City's Citi Bike system. Angels are users who earn points and rewards for helping to rebalance the system. The authors analysed the Bike Angels program and proposed several offline and online incentivizing policies, one of which has been adopted by Citi Bike.

O'Mahony [29] designed a raffle-based incentive system in which users can gain tickets for taking bikes from surplus stations and returning them at shortage stations. According to [30], this type of incentive is more effective than micro-incentives (e.g., smal discounts). 
Fricker and Gast [31] studied homogeneous BSSs and calculated the optimal fleet size to minimise the proportion of problematic stations (empty or full). They concluded that simply returning bikes to non-saturated stations does not produce a sufficient impact on the system behaviour. However, the mere incentive of suggesting users to return bikes at the "worst" among (only) two stations (chosen at random) improves the system dramatically, even if only some of the users follow the recommendations.

Chiariotti et al. [32] proposed a solution that combines rebalancing (with trucks) and incentivising users with rewards and prizes, as this combination has a lower cost than using trucks alone. Using a finite Markov birth-death process, their approach was to minimise the average expected time for which stations are empty or full. The authors were only interested in the overall effect of incentives on the system, rather than the effects of specific types of incentives.

$\mathrm{Li}$ and Shan [33] proposed a bidirectional incentive model where two types of users are considered-namely, commuters and leisure travellers. The users' travel behaviours are characterised as peak, flat peak, or inverse peak travel. The goal of this system is to persuade commuters to move from peak to flat peak behaviour, and leisure travellers from flat peak to inverse peak. This change in behaviour is incentivised by charging more to commuters or rewarding leisure users, respectively. Subsidies from the government are also included in their strategies.

User behaviour models are also relevant to static rebalancing approaches [22]. In [22], the behaviour of users when arriving at empty stations was simulated, i.e., whether they leave the system or walk to another station. Based on a survey, the authors calculated the rate of users who are willing to walk to a neighbouring station, depending on the existing distance.

In the present work, we do not focus on explicitly incentivizing users, but assume that they follow the recommendations made. As users gain experience with our recommendation service, we expect them to learn that it helps them to achieve a better user experience and to establish trust that the suggestions made are aligned with their goals. In this respect, our work is similar to [34], the authors of which created Cityride-a bike-sharing journey advisor for the city of Dublin. In their system, origin and destination stations are recommended based on the overall travel time and the probability of finding a bike at origin and a slot at destination stations at the respective expected arrival times. Even though rebalancing the system is not the primary goal of the recommendations, this is achieved indirectly as a side effect.

With respect to the applied mathematical tools and methods, our approach is related to the work by Waserhole and Jost [35], who theoretically studied the regulation of station-based vehicle-sharing systems through pricing using queuing networks. However, their study makes a series of assumptions that hinder its applicability-namely, null transportation times, infinite capacities of stations, and stationary demand over time.

As in the present paper, López Santiago et al. [36] also used historical data from the BiciMAD BSS in Madrid. They used social simulations to analyse the impact of several simple price incentive schemes and compare them to the policy currently employed in the BiciMAD BSS. Their work mainly focused on adequately modelling expected user behaviour and, in particular, the willingness to participate in rebalancing for given distances and incentives, based on existing surveys. In contrast, in the present work, we aim at systematically determining efficient solutions based on queuing theory as the basis for recommendations.

\section{Station-Based BSSs}

A station-based BSS consists of a set of stations distributed around the city at different, fixed geographical locations. Each station has a number of slots to park a bike (its capacity), which may differ from station to station. At a given point in time, some slots may be empty or available (e.g., can be used to return a bike), while others have an available bike plugged in that can be taken by a user. The total sum of bikes in the system is always much 
smaller than the sum of capacities of all stations, so as to allow users to find empty slots to return bikes.

Users who want to use the system would go to a station, take a bike, and return it after some time at another station close to the location of their destination. Normally, when a user decides to take a bike, they would be interested in finding the one closest to their initial position. In the same way, when they want to return a rented bike, they would like to do that at an available slot as close as possible to their final destination.

Usually, BSSs are supported by software applications that provide the users with realtime information about the current state of stations. Users may consult these applications in order to find appropriate stations to rent or to return a bike. In some cases, recommendation systems exist that propose appropriate stations to users. However, even if a user applies this real-time information to identify appropriate stations with available bikes or slots, it may occur that when they actually arrive at that station, the initially available bikes (or slots) have already been taken (or occupied) by other users. We call this situation an unsuccessful rental (or return) attempt. If a user has arrived at a station and there are no bikes available, they might consider going to a neighbouring station and trying there, or they may desist. A user will usually desist if they do not find an available bike after walking a certain distance (and perhaps trying at several stations). They might also desist if, based on the real-time information provided by their software application, they realize that there are currently no bikes available within a reasonable distance of their initial position. Once a user has rented a bike, they are obliged to return it at some station-that is, there is no possibility of desisting; the user must go to another station if there are no available slots at the first station they tried.

In this work, we intend to provide users with a recommendation system that suggests the "best" station to them when they want to rent a bike in the system (or they have to return a rented bike) - that is, we assume that potential users, when they decide to rent a bike or to return a rented bike, consult our system for recommendations of "good" stations. Furthermore, users would usually follow the given recommendation and move to a recommended station. In this sense, our aim in this paper is to define and analyse recommendation strategies that, if followed by the users, provide improved performance at both the individual and social (system) levels. The general objective is to recommend stations that (1) are likely to have the requested resource (bike or slot) once a user arrives there, and (2) provide short travel times, e.g., are close to the location of the user in case of a bike rental, or close to their final destination in case of a bike return.

In the rest of the paper, we use the following notations (see Table 1): $S=\left\{s_{1}, \ldots, s_{n}\right\}$ denotes the set of stations, each with geographical location $l\left(s_{i}\right)$ and capacity $c\left(s_{i}\right)$. bikes $\left(s_{i}, t\right)$ and $\operatorname{slots}\left(s_{i}, t\right)$ represent the number of available bikes and slots, respectively, at station $s_{i}$ at time $t$. A user $u_{k}$ who wishes to rent out a bike will request a recommendation for an appropriate rental station. Such a request, denoted by rent $t_{k}=\left\langle l_{k}, t, m d\right\rangle$, consists of the current (or desired origin) location $l_{k}$ of the user, the current time $t$ when the request is issued, and the maximum distance $m d$ that a user would be willing to walk to get a bike. If there is no station within distance $m d$, the user will desist and abandon the system. In a similar way, a user who wishes to return a rented bike will request a recommendation for a station to return that bike. This request is denoted by return $n_{k}=\left\langle l_{k}, d_{k}, t\right\rangle$, and consists of the current location $l_{k}$, the issuing time $t$, and the location of the user's final destination $d_{k}$. In the case of a return request, a maximum distance does not apply, since the user is required to return the bike in any case. wtime $(x, y)$ and $\operatorname{btime}(x, y)$ denote the expected time and wdist $(x, y)$, and bdist $(x, y)$-the expected distance to walk/cycle from location $x$ to location $y$. Both time and distance measures depend on the walking and cycling velocities of each user, and we assume that they can be estimated using standard velocities. 
Table 1. List of symbols and functions used.

\begin{tabular}{|c|c|}
\hline Symbols & Description \\
\hline$S=\left\{s_{1}, \ldots, s_{n}\right\}$ & Set of stations \\
\hline$l\left(s_{i}\right)$ & Location of station $s_{i}$ \\
\hline$c\left(s_{i}\right)$ & Capacity of station $s_{i}$ \\
\hline bikes $\left(s_{i}, t\right)$ & Number of available bikes at time $t$ \\
\hline $\operatorname{slots}\left(s_{i}, t\right)$ & Number of empty slots at station $s_{i}$ at time $t$ \\
\hline$u_{k}$ & User $k$ \\
\hline $\operatorname{rent}_{k}=\left\langle l_{k}, t, m d\right\rangle$ & $\begin{array}{l}\text { Rental request of user } k \text {, with: } l_{k} \text { (initial user location), } t \text { (time the request is issued), } m d \\
\text { (maximum distance the user is willing to walk to get a bike) }\end{array}$ \\
\hline return $_{k}=\left\langle l_{k}, d_{k}, t\right\rangle$ & $\begin{array}{l}\text { Return request of user } k \text {, with: } l_{k} \text { (initial user location), } d_{k} \text { (location of the user's final destination), } \\
t \text { (time the request is issued) }\end{array}$ \\
\hline wtime $(x, y)$ & Expected walking time from $x$ to $y$ \\
\hline $\operatorname{btime}(x, y)$ & Expected cycling time; from $x$ to $y$ \\
\hline wdist $(x, y)$ & Expected walking distance from $x$ to $y$ \\
\hline$b \operatorname{dist}(x, y)$ & Expected cycling distance from $x$ to $y$ \\
\hline RentStation $\left(\right.$ rent $\left._{k}\right)$ & Ordered sequence of recommended rental stations $\left(s_{1}, \ldots, s_{m}\right)=\left(s_{i}\right)_{i=1}^{m}$ \\
\hline ReturnStation $\left(\right.$ return $\left._{k}\right)$ & Ordered sequence of recommended return stations $\left(s_{1}, \ldots, s_{m}\right)=\left(s_{i}\right)_{i=1}^{m^{i}}$ \\
\hline$t_{\text {exp }}$ & Expected arrival time of a user at a station \\
\hline$t_{x}$ & Time of the last known expected event \\
\hline changes $\left(s_{i}, t, t_{\text {exp }}\right)$ & $\begin{array}{l}\text { Expected change in the number of bikes at station } s_{i} \text { in interval }\left[t, t_{\text {exp }}\right] \text { with respect to the number } \\
\text { of bikes at time } t\left(\text { e.g., }+2 \text { indicates there will be two more bikes at time } t_{\text {exp }}\right)\end{array}$ \\
\hline committedSlots $\left(s_{i}, t\right)$ & Maximum number of "committed" slots at station $s_{i}$ after time $t$. This value is $\geq 0$ \\
\hline committedBikes $\left(s_{i}, t\right)$ & Maximum number of "committed" bikes at station $s_{i}$ after time $t$. This value is $\geq 0$ \\
\hline estimatedBikes $\left(s_{i}\right.$, rent $\left._{k}\right)$ & $\begin{array}{l}\text { Number of expected available bikes at } s_{i} \text { at the time user } k \text { is expected to arrive and taking into } \\
\text { account the expected changes and "committed" bikes }\end{array}$ \\
\hline estimatedSlots $\left(s_{i}\right.$, return $\left._{k}\right)$ & $\begin{array}{l}\text { Number of expected available slots at } s_{i} \text { at the time user } k \text { is expected to arrive and taking into } \\
\text { account the expected changes and "committed" slots }\end{array}$ \\
\hline
\end{tabular}

We define rental and return recommendations as functions that map a rental or return request $\left(\right.$ rent $_{k}=\left\langle l_{k}, t, m d\right\rangle$ or return $\left.k_{k}=\left\langle l_{k}, d_{k}, t\right\rangle\right)$ to a ranking of stations (e.g., an ordered list with distinct pairwise elements): RentStation $\left(\right.$ rent $\left._{k}\right)=\left(s_{1}, \ldots, s_{m}\right)=\left(s_{i}\right)_{i=1}^{m}$ and ReturnStation $\left(\right.$ return $\left._{k}\right)=\left(s_{1}, \ldots, s_{m}\right)=\left(s_{i}\right)_{i=1}^{m}$ with $s_{i} \in S$.

\section{User-Centred Station Recommendation}

In this section, we define several strategies for recommending the best stations for renting/returning a bike from a user's perspective. We first present simple, straightforward versions of such user-centred recommendation strategies as baselines, and then develop a smarter strategy that forecasts expected changes in order to improve the estimation of available bikes/slots for the time when a user will actually arrive at a station. Finally, we present an even more sophisticated strategy that estimates the probability of finding available bikes/slots based on current demand data and uses this information to calculate a "cost" for renting or returning a bike at a station.

\subsection{Standard Strategies}

\subsubsection{Shortest Distance}

This is a basic selection strategy, where a user simply prefers the station that is closest to their origin/destination position. This strategy represents the behaviour of a user who just goes to the closest station, without knowing whether there are available bikes or slots:

$$
\operatorname{RentStation}\left(\text { rent }_{k}\right)=\left(s_{i}\right)_{i=1}^{m}
$$

where:

$$
\begin{gathered}
\forall s_{i}: \operatorname{wdist}\left(l_{k}, l\left(s_{i}\right)\right) \leq m d \wedge \forall s_{i}, s_{j}, i<j: \operatorname{wdist}\left(l_{k}, l\left(s_{i}\right)\right) \leq w \operatorname{wist}\left(l_{k}, l\left(s_{j}\right)\right) \\
\text { ReturnStation }\left(\text { return }_{k}\right)=\left(s_{i}\right)_{i=1}^{m}
\end{gathered}
$$


where:

$$
\forall s_{i}, s_{j}, i<j: \operatorname{wdist}\left(l\left(s_{i}\right), d_{k}\right) \leq \operatorname{wdist}\left(l\left(s_{j}\right), d_{k}\right)
$$

\subsubsection{Informed Shortest Distance}

In this strategy, the system proposes the closest stations that have available bikes (or slots). Thus, a user avoids going to stations that are empty (at the moment of station selection). However, a station may no longer hold available bikes or slots at the time when the user actually arrives at it, because other users have taken the available bikes (or have occupied the available slots):

$$
\operatorname{RentStation}\left(\text { rent }_{k}\right)=\left(s_{i}\right)_{i=1}^{m}
$$

where:

$$
\begin{gathered}
\forall s_{i}: \operatorname{wdist}\left(l_{k}, l\left(s_{i}\right)\right) \leq m d \wedge \operatorname{bikes}\left(s_{i}, t\right)>0 \wedge \\
\forall s_{i}, s_{j}, i<j: \operatorname{wdist}\left(l_{k}, l\left(s_{i}\right)\right) \leq \operatorname{wdist}\left(l_{k}, l\left(s_{j}\right)\right) \\
\operatorname{ReturnStation}\left(\operatorname{return}_{k}\right)=\left(s_{i}\right)_{i=1}^{m}
\end{gathered}
$$

where:

$$
\forall s_{i}: \operatorname{slots}\left(s_{i}, t\right)>0 \wedge \forall s_{i}, s_{j}, i<j: \operatorname{wdist}\left(l\left(s_{i}\right), d_{k}\right) \leq w \operatorname{wist}\left(l\left(s_{i}\right), d_{k}\right)
$$

\subsubsection{Distance Resources}

Here, stations are selected by combining distance and available resources. The preferred stations are the ones that are closer to the origin (or destination) location of the user and have more available bikes (slots) at the time a recommendation is requested:

$$
\operatorname{RentStation}\left(\text { rent }_{k}\right)=\left(s_{i}\right)_{i=1}^{m}
$$

where:

$$
\begin{gathered}
\forall s_{i}: \text { wdist }\left(l_{k}, l\left(s_{i}\right)\right) \leq m d \wedge \operatorname{bikes}\left(s_{i}, t\right)>0 \wedge \\
\forall s_{i}, s_{j}, i<j: \frac{\operatorname{wdist}\left(l_{k}, l\left(s_{i}\right)\right)}{\operatorname{bikes}\left(s_{i}, t\right)} \leq \frac{\operatorname{wdist}\left(l_{k}, l\left(s_{j}\right)\right)}{\operatorname{bikes}\left(s_{j}, t\right)} \\
\text { ReturnStation }\left(\text { return }_{k}\right)=\left(s_{i}\right)_{i=1}^{m}
\end{gathered}
$$

where:

$$
\forall s_{i}: \operatorname{slots}\left(s_{i}, t\right)>0 \wedge \forall s_{i}, s_{j}, i<j: \frac{w \operatorname{dist}\left(l\left(s_{i}\right), d_{k}\right)}{\operatorname{slots}\left(s_{i}, t\right)} \leq \frac{w \operatorname{dist}\left(l\left(s_{j}\right), d_{k}\right)}{\operatorname{slots}\left(s_{j}, t\right)}
$$

\subsection{Distance Expected Resources}

If a recommendation or information system is used by users to select a station to rent or return a bike, this system could take into account past requests in order to make better estimations of available bikes at a station in the near future. In particular, if a user $u_{k}$ asks at time $t$ to rent or return a bike, the system may recommend a station $s_{i}$, and can assume that the user will take or return a bike there when they arrive, i.e., at time $t+w \operatorname{time}\left(l_{k}, l\left(s_{i}\right)\right)$ or $t+\operatorname{btime}\left(l_{k}, l\left(s_{i}\right)\right)$, respectively. This information can be used to better determine whether or not there will be available bikes at $s_{i}$ if another user requests information. In the same way, expected returns of bikes can help to predict available slots.

Figure 1 explains this idea in more detail. Suppose that at time $t$, user $u_{k}$ (at location $l_{k}$ ) asks for a station to rent a bike, and the given option would be station $s_{i}$. At time $t$, there are $n$ available bikes at station $s_{i}$, and the system tries to estimate the available bikes at the moment when $u_{k}$ would actually arrive at $s_{i}$, i.e., at time $t_{\text {exp }}=t+w$ time $\left(l_{k}, l\left(s_{i}\right)\right)$. Before instant $t$, the system has already recommended station $s_{i}$ to other users for either renting or returning bikes, and assumes that those users will actually follow the recommendations and, thus, will return or take bikes at their expected arrival times. These actions are reflected 
in Figure 1: at time $t_{1}$, a bike is expected to be taken; at $t_{2}$, a bike is expected to be returned, and so on. Let changes $\left(s_{i}, t, t_{\text {exp }}\right)$ denote the sum of the expected changes in the number of available bikes at station $s_{i}$ in the time interval between the time of issuing a user request $t$ and the potential arrival of the user at $t_{\text {exp }}$. Here, each bike rental in this period counts as -1 , and each bike return counts as +1 . In the example, changes $\left(s_{i}, t, t_{\text {exp }}\right)$ would be -1 . We can now estimate the number of bikes or slots expected to be available at the arrival time $t_{\text {exp }}$ of user $u_{k}$ by bikes $\left(s_{i}, t\right)+\operatorname{changes}\left(s_{i}, t, t_{\text {exp }}\right)$ and $\operatorname{slots}\left(s_{i}, t\right)-\operatorname{changes}\left(s_{i}, t, t_{\text {exp }}\right)$.

\section{Evolution of bikes at station si}

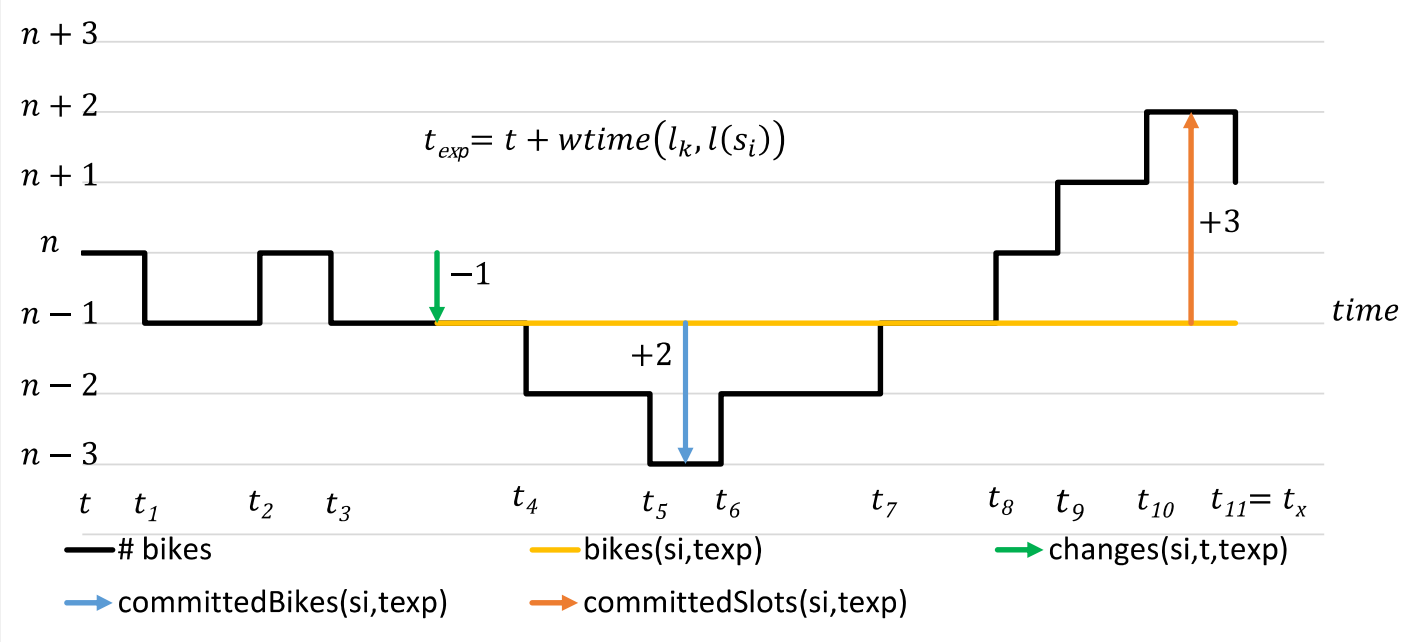

Figure 1. Example of the evolution of the number of bikes at a station. Values of changes $\left(s_{i}, t, t_{\text {exp }}\right), \operatorname{commitedBikes}\left(s_{i}, t_{\text {exp }}\right)$, and commitedSlots $\left(s_{i}, t_{\exp }\right)$ are shown.

The system may also take into account the possibility that even if there are some bikes or slots available when user $u_{k}$ arrives, some of those may have been already "committed", e.g., they have been the basis for recommendations to other users who will eventually arrive after the expected arrival time of user $u_{k}$. In Figure 1, this refers to the events at $t_{4}$, $t_{5}, \ldots t_{x}$. Such events could be conceived as "implicit reservations", and the system could try to "block" a number of bikes or slots for further recommendations. Here, we do not refer to a real blocking of a bike or slot for future users. Instead, the system would simply factor in this information when determining recommendations.

The number of "committed" bikes or slots depends on the sequence of expected events, and corresponds to the worst cases: the maximum and minimum values of changes $\left(s_{i}, t_{\text {exp }}, t_{x}\right)$, the expected changes after the user arrives, up to the last known event at time $t_{x}$ (or $t_{x}=t_{\text {exp }}$, if there is none). We denote these two values by:

$$
\operatorname{committedSlots}\left(s_{i}, t_{\text {exp }}\right)=\max _{t_{\exp } \leq t_{i} \leq t_{x}}\left(\operatorname{changes}\left(s_{i}, t_{\text {exp }}, t_{i}\right)\right)
$$

and:

$$
\operatorname{committedBikes}\left(s_{i}, t_{\text {exp }}\right)=\left|\min _{t_{\exp } \leq t_{i} \leq t_{x}}\left(\operatorname{changes}\left(s_{i}, t_{\text {exp }}, t_{i}\right)\right)\right|
$$

In Figure 1, commitedSlots $\left(s_{i}, t_{\text {exp }}\right)=3$ signifies that three extra slots have to be "reserved" because a sequence of two taken and five returned bikes reaches a peak of 3 before $t_{x}$. On the other hand, commitedBikes $\left(s_{i}, t_{\text {exp }}\right)=2$ indicates that, at some point (in this case at $\left.t_{5}\right)$, two extra bikes will be taken from the station.

Taking into account the changes expected to occur at a station $s_{i}$ after $t$ and before the expected arrival of user $u_{k}$, as well as the "committed" bikes and slots, the estimated 
available bikes and slots at station $s_{i}$ for a rental and a return request rent $t_{k}=\left\langle l_{k}, t, m d\right\rangle$ and return $_{k}=\left\langle l_{k}, d_{k}, t\right\rangle$ are defined as:

$$
\begin{aligned}
& \text { estimatedBikes }\left(s_{i}, \text { rent }_{k}\right) \\
& =\operatorname{bikes}\left(s_{i}, t\right)+\operatorname{changes}\left(s_{i}, t, t_{\text {exp }}\right) \\
& \text { - commitedBikes }\left(s_{i}, t_{\text {exp }}\right) \\
& \text { estimatedSlots }\left(s_{i}, \text { return }_{k}\right) \\
& =\operatorname{slots}\left(s_{i}, t\right)-\operatorname{changes}\left(s_{i}, t, t_{\text {exp }}\right) \\
& \text { - commitedSlots }\left(s_{i}, t_{\text {exp }}\right)
\end{aligned}
$$

where $t_{\text {exp }}$ is the estimated expected arrival time of user $u_{k}$ at $s_{i}$.

With these definitions, we can define the DistanceExpectedResources strategy as follows:

$$
\text { RentStation }_{\left(\text {rent }_{k}\right)}=\left(s_{i}\right)_{i=1}^{m}
$$

where:

$$
\begin{gathered}
\forall s_{i}: \text { wdist }\left(l_{k}, l\left(s_{i}\right)\right) \leq m \wedge \wedge \text { estimatedBikes }\left(s_{i}, \text { rent }_{k}\right)>0 \wedge \\
\forall s_{i}, s_{j}, i<j: \frac{\text { wdist }\left(l_{k}, l\left(s_{i}\right)\right)}{\text { estimatedBikes }\left(s_{i}, \text { rent }_{k}\right)} \leq \frac{\text { wdist }\left(l_{k}, l\left(s_{j}\right)\right)}{\text { estimatedBikes }\left(s_{j}, \text { rent }_{k}\right)} \\
\text { ReturnStation }\left(\text { return }_{k}\right)=\left(s_{i}\right)_{i=1}^{m}
\end{gathered}
$$

where:

$$
\begin{aligned}
& \forall s_{i}: \text { estimatedSlots }\left(s_{i}, \text { return }_{k}\right)>0 \wedge \\
& \forall s_{i}, s_{j}, i<j: \frac{\left.w d i s t\left(l\left(s_{i}\right), d_{k}\right)\right)}{\text { estimatedSlots }\left(s_{i}, \text { return }_{k}\right)} \leq \frac{\operatorname{wdist}\left(l\left(s_{j}\right), d_{k}\right)}{\text { estimatedSlots }\left(s_{j}, \text { return }_{k}\right)}
\end{aligned}
$$

\begin{tabular}{|c|c|}
\hline Symbols & Description \\
\hline $\operatorname{rentDemand}\left(s_{i}, t_{a}, t_{b}\right)$ & Number of expected rent attempts at $s_{i}$ between $t_{a}$ and $t_{b}$ \\
\hline returnDemand $\left(s_{i}, t_{a}, t_{b}\right)$ & Number of expected return attempts at $s_{i}$ between $t_{a}$ and $t_{b}$ \\
\hline$\pi^{i}(t)$ & (Transient) state probability distribution of the number of bikes at station $s_{i}$ at time $t$ \\
\hline$\pi_{j}^{i}(t)$ & Probability that there are $j$ available bikes at station $s_{i}$ at time $t$ \\
\hline bikeProb $\left(s_{i}\right.$, rent $\left._{k}\right)$ & Probability that $u_{k}$ would find an available bike at station $s_{i}$ when they arrive \\
\hline $\operatorname{slotProb}\left(s_{i}\right.$, return $\left._{k}\right)$ & Probability that $u_{k}$ would find an available slot at station $s_{i}$ when they arrive \\
\hline$\lambda$ & Average bike arrival rate \\
\hline$\mu$ & Average bike rental rate \\
\hline RentFailCost & Cost of getting to a station and finding no available bike \\
\hline ReturnFailCost & Cost of getting to a station and finding no available slot \\
\hline localRentCost $\left(s_{i}\right.$, rent $_{k}$, RentFailCost $)$ & $\begin{array}{l}\text { Estimated cost for user } u_{k} \text { to rent a bike at } s_{i} \text {, resulting from combining the walking time to } \\
\text { the station and the probability of finding an available bike }\end{array}$ \\
\hline localReturnCost $\left(s_{i}\right.$, return $_{k}$, ReturnFailCost $)$ & $\begin{array}{l}\text { Estimated cost for user } u_{k} \text { to return a bike at } s_{i} \text {, resulting from combining the cycling time } \\
\text { to the station, walking time to the final destination, and the probability of finding an } \\
\text { available slot }\end{array}$ \\
\hline
\end{tabular}

\subsection{Expected Cost}

It seems obvious that a better estimation of the available bikes or slots at the time of a user's arrival at a station would prevent unsuccessful rental or return attempts. One way to achieve this is by estimating the probability distributions of available bikes at a station at a future instance in time, based on known demand patterns. In the following section, we describe how demands can be estimated, how we estimate the probabilities of finding an available bike/slot at a station at any time in the future, and how we use such probabilities to rank stations increasingly based on cost, combining the probability of finding a bike/slot and the expected time to reach a station.

Table 2 summarises the symbols and functions introduced in this section.

Table 2. List of symbols and functions used. 


\subsubsection{Demand Estimation}

The demand for bike rentals and returns at a given station can be estimated from historical data and for different time intervals, days, weather conditions, etc. Supposing that such data are available, we use rentDemand $\left(s_{i}, t_{a}, t_{b}\right)$ and returnDemand $\left(s_{i}, t_{a}, t_{b}\right)$ to reflect the number of expected rental and return attempts at station $s_{i}$ during the time interval between $t_{a}$ and $t_{b}\left(t_{a}<t_{b}\right)$. Given sufficient historical data, the calculation of these values is straightforward and, as such, we omit it here.

\subsubsection{Probability Calculation}

Using queueing theory, a station for renting bikes can be modelled as an $\mathrm{M} / \mathrm{M} / 1 / \mathrm{K}$ queue (see, e.g., $[21,31,35]$. We consider that bikes arrive at a station and request the "service" to be rented. The arrival process (e.g., the return of bikes through users) and the "service" process (e.g., the rental of bikes by users) follow a Poisson distribution. The number of service channels is 1 and the maximum number of bikes waiting to be rented is $\mathrm{K}$ (the capacity of the station). The $\mathrm{M} / \mathrm{M} / 1 / \mathrm{K}$ queue is a special case of a birth-death process and of continuous-time Markov chains with $\mathrm{K}+1$ states. Let $\lambda$ be the average bike arrival rate and $\mu$ the average bike rental rate; given a station $s_{i}$, let $\pi^{i}(\mathrm{t})$ denote the (transient) state probability distribution and $\pi_{j}^{i}(t)$ the probability that the system is in state $j$ at time $t$-that is, $\pi_{j}^{i}(t)$ represents the probability that there are $j$ available bikes at station $s_{i}$ at time $t$.

The time-dependent behaviour of the probability distribution can be determined by the system of differential equations (known as the forward Kolmogorov equations):

$$
\begin{gathered}
\frac{d \pi_{0}^{i}(t)}{d t}=-\lambda \pi_{0}^{i}(t)+\mu \pi_{1}^{i}(t) \\
\frac{d \pi_{j}^{i}(t)}{d t}=\lambda \pi_{j-1}^{i}(t)+\mu \pi_{j+1}^{i}(t)-(\lambda+\mu) \pi_{j}^{i}(t), \text { for } 0<j<K \\
\frac{d \pi_{K}^{i}(t)}{d t}=\lambda \pi_{K-1}^{i}(t)-\mu \pi_{K}^{i}(t)
\end{gathered}
$$

Given an initial distribution $\pi^{i}(0)$ with $\sum_{j=0}^{K} \pi_{j}^{i}(, 0)=1$, the system can be solved, obtaining the distribution $\pi^{i}(\mathrm{t})$ at time $t$. Solving Equation (1) is mathematically complex, but an acceptable approximation can be found. In particular, in our experimental implementation, we use the fourth-order Runge-Kutta method for iteratively approximating solutions of differential equations (given an initial problem defined by $\frac{d y}{d t}=f(t, y)$, and $y\left(t_{0}\right)=y_{0}$, solutions for $y\left(t_{0}+\Delta t\right)$ are approximated by iteratively calculating $y\left(t_{i}+h\right)$ based on $y\left(t_{i}\right)$ and $t_{i}$. Taking small steps $h<\Delta t$, this process is repeated for $i$ steps, until $t_{i}+h$ is approximately $t_{0}+\Delta t$. In this case, $y\left(t_{i}+h\right)$ approximates $\left.y\left(t_{0}+\Delta t\right)\right)$.

Let rent $_{k}=\left\langle l_{k}, t, m d\right\rangle$ be a user rental request, $s_{i}$ a potential station, and $t_{\text {exp }}=t+$ wtime $\left(l_{k}, l\left(s_{i}\right)\right)$ the expected arrival time of user $u_{k}$ at station $s_{i}$. Then, Equations (13)-(15) can be used to calculate the probability that $u_{k}$ would find an available bike (or slot) at station $s_{i}$ when they arrive at time $t_{\text {exp }}$. These probabilities are denoted as bikeProb $\left(s_{i}\right.$, rent $\left._{k}\right)$ and slotProb $\left(s_{i}, \operatorname{return}_{k}\right)$, respectively.

We define $\pi^{i}(0)$, the current probability distribution of (available) bikes locked at station $s_{i}$ and the current time $t$, by:

$$
\pi_{j}^{i}(0)=\left\{\begin{array}{c}
1, \text { if } j=\operatorname{bikes}\left(s_{i}, t\right)+\operatorname{changes}\left(s_{i}, t, t_{\text {exp }}\right) \\
0, \text { otherwise }
\end{array}\right.
$$

Note that, similarly to the DistanceExpectedResources strategy defined before, we include changes in the number of available bikes at the station that are expected to take place 
between $t$ and $t_{\text {exp }}$. In particular, we approach such changes as if they had already been "blocked" at the moment of the user request $(t)$.

Using $\pi^{i}(0)$ as described above, setting $\lambda=\operatorname{returnDemand}\left(s_{i}, t, t_{\text {exp }}\right)$ and $\mu=$ rentDemand $\left(s_{i}, t, t_{\text {exp }}\right)$, and solving the system in the sense of Equations (13)-(15), we can approximate a solution for the probability distribution of available bikes at the time when the user arrives at the station: $\pi^{i}\left(t_{\exp }\right)$; meanwhile, $\pi^{i}\left(t_{\exp }\right)$ can be used to calculate the probability that user $u_{k}$ will find a bike when they arrive at station $s_{i}$ (at expected arrival time $\left.t_{\text {exp }}\right)$ :

$$
\operatorname{bikeProb}\left(s_{i}, \text { rent }_{k}\right)=\sum_{j=1+\text { committedBikes }\left(s_{i}, t_{\text {exp }}\right)}^{c\left(s_{i}\right)} \pi_{j}^{i}\left(t_{\text {exp }}\right)
$$

As defined previously, comittedBikes $\left(s_{i}, t_{\exp }\right)$ represents the maximum number of bikes that are "committed", e.g., that should be "blocked" for other users already known to arrive at station $s_{i}$. Thus, bikeProb $\left(s_{i}\right.$, rent $\left._{k}\right)$ is not just the probability that a given user $u_{k}$ will find an available bike at a station when they arrive, but the probability of having enough bikes for the user as well as all other users who have been recommended to rent a bike at this station and are expected to arrive after user $u_{k}$. For instance, if there are a maximum of two "committed" bikes, bikeProb $\left(s_{i}\right.$, rent $\left._{k}\right)$ would be the probability that the number of available bikes at the expected arrival time $t_{\text {exp }}$ is three or more.

In a similar way, in the case of a return request return $_{k}$, we must estimate the probability that, at the expected arrival time $t_{\text {exp }}$, there is at least one slot available, plus the maximum required number of "committed" slots for future expected users. Considering the capacity of a station $\left(c\left(s_{i}\right)\right)$, the probability of having at least $1+\operatorname{committedSlots}\left(s_{i}, t_{\exp }\right)$ slots is equivalent to having at most $c\left(s_{i}\right)-\left(1+\operatorname{committedSlots}\left(s_{i}, t_{\text {exp }}\right)\right)$ locked bikes. Thus, we calculate the return probability by:

$$
\operatorname{slotProb}\left(s_{i}, \operatorname{return}_{k}\right)=\sum_{j=0}^{c\left(s_{i}\right)-\left(1+\operatorname{committedSlots}\left(s_{i}, t_{\text {exp }}\right)\right)} \pi_{j}^{i}\left(t_{\text {exp }}\right)
$$

\subsubsection{Expected Cost Recommendation}

We combine distance and bike or slot probabilities to define the (local) rental or return cost of a station $s_{i}$ for a user $u_{k}$ :

$$
\begin{aligned}
& \operatorname{localRentCost}\left(s_{i}, \text { rent }_{k}, \operatorname{RentFailCost}\right) \\
& =w_{\text {time }}\left(l_{k}, l\left(s_{i}\right)\right)+\left(1-\operatorname{bikeProb}\left(s_{i}, \text { rent }_{k}\right)\right) \\
& \cdot \operatorname{RentFailCost}
\end{aligned}
$$

$$
\begin{aligned}
\text { localReturnCost } & \left(s_{i}, \text { return }_{k}, \text { ReturnFailCost }\right) \\
& \left.=\operatorname{btime}_{(}, l\left(s_{i}\right)\right)+\operatorname{slotProb}\left(s_{i}, \operatorname{return}_{k}\right) \cdot \operatorname{wtime}\left(l\left(s_{i}\right), d_{k}\right) \\
& +\left(1-\operatorname{slotProb}\left(s_{i}, \operatorname{return}_{k}\right)\right) \cdot \operatorname{ReturnFailCost}
\end{aligned}
$$

RentFailCost and ReturnFailCost are parameters that represent the associated cost of getting to a station and finding no available bike or slot, respectively. In order to avoid distortions, in case wtime $\left(l\left(s_{i}\right), d_{k}\right)>$ ReturnFailCost, we set localReturnCost $\left(s_{i}\right.$, return $_{k}$, ReturnFailCost $)=\operatorname{btime}\left(l_{k}, l\left(s_{i}\right)\right)+\operatorname{wtime}\left(l\left(s_{i}\right), d_{k}\right)$. It should also be noted that in the return cost we take into account not only the walking time from the station to the final destination, but also the cycling time to the selected station.

Using the cost functions, the definition of the Expected Cost recommendation strategy simply orders stations by increasing cost:

$$
\text { entStation }\left(\text { rent }_{k}\right)=\left(s_{i}\right)_{i=1}^{m}
$$


where:

$$
\begin{aligned}
& \forall s_{i}: \text { wdist }\left(l_{k}, l\left(s_{i}\right)\right) \leq m d \wedge \\
& \begin{array}{c}
\forall s_{i}, s_{j}, i<j: \text { localRentCost }\left(s_{i}, \text { rent }_{k}, \text { RentFailCost }\right) \\
\leq \text { localRentCost }\left(s_{j}, \text { rent }_{k}, \text { RentFailCost }\right)
\end{array} \\
& \text { ReturnStation }\left(\text { return }_{k}\right)=\left(s_{i}\right)_{i=1}^{m}
\end{aligned}
$$

where:

$$
\begin{aligned}
\forall s_{i}, s_{j}, i<j: \text { localReturnCost }\left(s_{i}, \text { return }_{k}, \text { ReturnFailCost }\right) \\
\quad \leq \text { localReturn } \operatorname{Cost}\left(s_{j}, \text { return }_{k}, \text { ReturnFailCost }\right)
\end{aligned}
$$

\section{Evaluation of User-Centred Recommendation}

We used the Bike3S simulation tool [37] to validate the proposed strategies and compare their performance to the standard strategies. Bike3S is intended to evaluate different rebalancing strategies. The simulated infrastructure includes the location, capacity, and available bikes for each station. During a simulation, users are generated and interact with the infrastructure to rent or return bikes. They may ask a service (strategy) for a recommendation to choose their target station. Several user models can be defined and used in the same simulation run. Users' appearance time, initial location, and destination are loaded into the simulator. A tool facilitates the creation of this information randomly. Different strategies can be implemented and easily integrated into Bike3S.

We replicated the operation of the BiciMAD public bike-sharing system in Madrid (Spain). This system covers an area of approximately $5 \times 5 \mathrm{~km}$ of central Madrid, and is continuously growing. Currently it utilises $\sim 200$ stations and 2500 bicycles. The capacity of the stations is between 12 and 30 (most stations have 24 slots).

\subsection{Simulation Experiment Setup}

There are publicly available data on the usage of the BiciMAD system, in particular:

- Data on the trips: Including, for each trip, the time of taking a bike, origin station, destination station, travel time, and approximate route. However, in order to anonymize the data, only the day and hour of the pick-up time of each trip are given (without minutes). Each trip includes a user type, with possible values representing regular or occasional users, BiciMAD staff, or unidentifiable users;

- Situation of the stations: including the number of available bikes and slots, and whether or not a station was active.

In order to replicate a real-world scenario, we extracted the user data for a 24-h period (in particular, from 7:00 on 20 July 2018 to 7:00 on 21 July 2018). That period comprised 12,296 real user trips, whose data were used to generate the artificial users of our simulation. These data, however, only include the stations where a user took or returned a bike, but not their origin or destination location. Therefore, and in order to reflect the real situation as accurately as possible, we generated random origin and destination locations in an area spanned by a circle of $300 \mathrm{~m}$ around the rental station and the return station, respectively. Finally, the instant of creation of the simulated user was generated randomly within the hour of their appearance in the real-world data (with a uniform distribution), since the exact minute and second of appearance were unknown.

For specifying the initial station configuration in the simulated scenario, we used the official (real) data at the initial time (20 July 2018 at 7:00), at which there were 169 active stations with a total capacity of 4086 slots and 1792 bikes plugged in. However, since we wanted to analyse the performance of the different recommendation strategies in rather critical situations (where it is more likely to have empty and full stations), we reduced the station capacities and the number of plugged-in bikes at each station to approximately half of the original values - that is, in our experiments, we used 169 stations with a total capacity of 1995 slots and 896 bikes. 
Even though the Bike3S tool can simulate the movements of people walking or cycling on real networks of roads and footpaths (using OpenStreetMap data), in our simulation, we used straight-line movements on the geographical map. The impact of this choice on the performance comparison of the analysed strategies was marginal, but it allowed us to reduce the simulation time considerably. We considered a default walking and cycling velocity of $1.4 \mathrm{~m} / \mathrm{s}$ and $4 \mathrm{~m} / \mathrm{s}$, respectively [38,39]. However, since we used straight-line movements, and in order to adjust to more realistic values, we applied a velocity factor of 0.614. This factor was established based on comparing real and straight-line travel times between a set of origin/destination locations in Madrid (using OpenStreetMap data). Thus, the actual applied velocities were $0.8596 \mathrm{~m} / \mathrm{s}$ for walking and $2.456 \mathrm{~m} / \mathrm{s}$ for cycling. These velocities were used to simulate the movements of users; they were also used in the recommendation strategies to estimate the expected arrival times of users.

Using the specified setup, we carried out experiments to compare the performance of the five previously defined recommendation strategies:

- $\quad$ ShortestDistance (SD);

- InformedShortestDistance (ISD);

- DistanceResources (DR);

- DistanceExpectedResources (DER);

- ExpectedCost $(E C(x, y))$, where $x=$ RentFailCost and $y=$ ReturnFailCost.

The user behaviour for renting a bike in the simulations was as follows:

1. A user $u_{k}$ appears at a geographical location $l_{k}$ at time $t$ and asks the recommendation system for a rental recommendation (with request rent $t_{k}=\left\langle l_{k}, t, m d\right\rangle$ ). The maximum acceptable distance in the experiments is set to $m d=600 \mathrm{~m}$;

2. The recommendation system applies its strategy and returns a ranking of possible stations;

3. Given the ranking, the user filters out all stations that they have already tried. If no stations are left, the user will abandon the system without renting a bike. Otherwise, they walk towards the first station in the list of recommendations in order to rent a bike;

4. In case the user gets to a station and there are no available bikes, they repeat the whole process until they either abandon the search or finally find a bike. In this case, the value of the maximum acceptable distance $(m d)$ is reduced by the distance the user has walked already.

With this behaviour, a user will effectively abandon the system if they do not find any bikes within $m d=600 \mathrm{~m}$.

For finding a return station, the process is as follows:

1. In the moment a user $u_{k}$ has rented a bike at a station $s_{i}$, they issue a return request return $n_{k}=\left\langle l_{k}, t, l_{d}\right\rangle$, where $l_{k}$ and $t$ are the current position and time, and $l_{d}$ is their final destination location;

2. The recommendation system returns a ranking of stations for returning the bike;

3. Given the ranking, the user filters out any stations that they have already tried, and selects the first remaining station for returning the bike;

4. In case the user gets to a station and there are no available slots, they repeat the whole process until they finally find a station to leave the bike (there is no possibility to abandon the attempt).

\subsection{Simulation Results}

The main aim of the recommendation strategies is to allow an efficient usage of a BSS in terms of the time the users spend to go from their origin location to their destination, as well as the unsuccessful rental and return attempts.

Table 3 compares the performance of the different user-centred recommendation strategies. The measurements we present are the following: 
- \#a: number of users who dropped out (abandoned) the system, and percentage with regards to users that finished;

- \#fh: number of failed user rental attempts and percentage over all user rental attempts;

- \#fr: number of failed user return attempts and percentage over all user return attempts;

- $t t$ : average total time of users in the system; this is based on the time a user requires to go from their origin to a bike rental station, to cycle from there to a station to return the bike and, finally, to walk to their final destination. The value is averaged over all users who were able to rent a bike (i.e., who did not abandon the attempt);

- $A E T$ : average station empty time; this is the time for which a station has been empty (without available bikes) and, thus, would potentially have been denying service. The value is the average over all 169 stations for the whole simulation period.

Table 3. Experimental results for Madrid. Bold numbers indicate the best obtained result for each metric.

\begin{tabular}{cccccc}
\hline Strategy & \#a/\% & \#fh/\% & \#fr $/ \%$ & tt (min) & AET (min) \\
\hline OPTIMUM & 0 & 0 & 0 & 19.21 & \\
\hline SD & $1573 / 12.85$ & $1834 / 14.67$ & $5148 / 32.6$ & 22.47 & 378.4 \\
\hline ISD & $895 / 7.3$ & $415 / 3.53$ & $1965 / 14.7$ & 21.86 & 398.1 \\
\hline DR & $461 / 3.76$ & $126 / 1.06$ & $225 / 1.9$ & 21.54 & 201.9 \\
\hline DER & $243 / 1.98$ & $\mathbf{0 / 0}$ & $\mathbf{0 / 0}$ & 21.62 & 189.7 \\
\hline EC $(1000 / 2000)$ & $428 / 3.49$ & $72 / 0.6$ & $269 / 2.2$ & $\mathbf{2 1 . 1 2}$ & 362.7 \\
\hline EC $(3000 / 2000)$ & $367 / 2.99$ & $13 / 0.11$ & $183 / 1.5$ & 21.24 & 309.0 \\
\hline EC $(70000 / 2000)$ & $240 / 1.96$ & $3 / 0.02$ & $260 / 2.1$ & 21.6 & 197.5 \\
\hline EC $\left(10^{6} / 10^{6}\right)$ & $\mathbf{1 4 8 / 1 . 2 1}$ & $1 / 0.01$ & $\mathbf{0 / 0}$ & 23.21 & $\mathbf{7 5 . 7}$ \\
\hline
\end{tabular}

The first row of Table 3 specifies a hypothetical optimal situation, where every user can obtain a bike at the station closest to their origin and return it at the station closest to their destination (i.e., the station capacities for rental and return are assumed to be unlimited). In this case, the average travel time would be $19.21 \mathrm{~min}$. This travel time can obviously not be obtained in a real scenario.

Analysing the standard strategies (the first four strategies in Table 3), there are different conclusions:

$S D$ has the worst behaviour. Here, users go to the closest station, and many users will abandon their search because there are no bikes available. Furthermore, the travel time is quite high, because after an unsuccessful rental or return attempt, users may try at other stations and, thus, increase the time that they spend in the system. In comparison, ISD has a much better performance, because the probability of finding an available bike or slot is much higher if a user only goes to the stations that have available resources at the moment of the recommendation request.

$D R$ combines the distance and availability of resources. In this way, it obtains a better dropout rate and, at the same time, a shorter travel time than SD and ISD. It should also be noted that, here, users would slightly prefer to rent/return bikes where there are more bikes or slots (within closed stations). This means, here, users implicitly improve the distribution of the bikes (and slots) among the stations. This can be seen in the reduction of the average empty time of stations.

The DistanceExpectedResources strategy (DER) clearly outperforms the standard strategies. Compared to $D R, D E R$ not only includes the bikes/slots in the analysis of appropriate stations that are available at the precise moment when a user issues a recommendation request, but also the expected changes up to the expected arrival of the user. In this case, the dropout rate can be reduced from $3.76 \%$ to $1.98 \%$, whereas the average travel time remains nearly the same (21.62 versus $21.54 \mathrm{~min}$ ). It should be noted that, with $D E R$, the failed rental and return attempts are both 0 . This is because with the specified experimental setting, the estimation of available bikes/slots is exact because (1) users always go to 
the recommended stations, and (2) the estimated travel time of users is the same as their actual travel time. In a real scenario, the benefit of $D E R$ with respect to $D R$ should be somewhat smaller.

The last four rows in Table 3 correspond to different instances of the ExpectedCost strategy $(E C)$, which prioritises stations with a lower cost, calculated based on travel time and the (demand-dependent) probability of finding a bike/slot at the expected time of arrival of the user. This strategy's performance varies for different values of RentFailCost and ReturnFailCost. In general, higher penalizations produce a lower abandonment rate, but increase the travel time. With RentFailCost $=$ ReturnFailCost $=1,000,000$, for example, the abandonment rate is reduced to $1.21 \%$, but the travel time increases to $23.21 \mathrm{~min}$, which is considerable. A rather high value for RentFailCost and low value for ReturnFailCost obtains a good compromise. In this sense, for RentFailCost $=70,000$ and ReturnFailCost $=2000$, both dropout rate and travel time can be slightly reduced with regard to $D E R$. With rather low penalizations, the abandonment rate is higher, but the travel time can be reduced, as with EC $(1000,2000)$.

In Figures 2-4, we analyse the behaviour of EC with varying values for RentFailCost and ReturnFailCost.

As shown in Figure 2, the abandonment rate decreases with increasing ReturnFailCost, and the travel time decreases up to a value of $\sim 2000$, and then increases. At higher values, both travel time and abandonment rate become almost stable. The lowest travel time is obtained with a value of 2000 . Exactly the same behaviour is observed for other values of RentFailCost. We also observed in our experiments that, as the value of ReturnFailCost increases, the number of failed returns (\#fr) falls rapidly towards $\sim 0$ at the value of ReturnFailCost $=4000$ (for the sake of clarity, this is not shown in the figure).

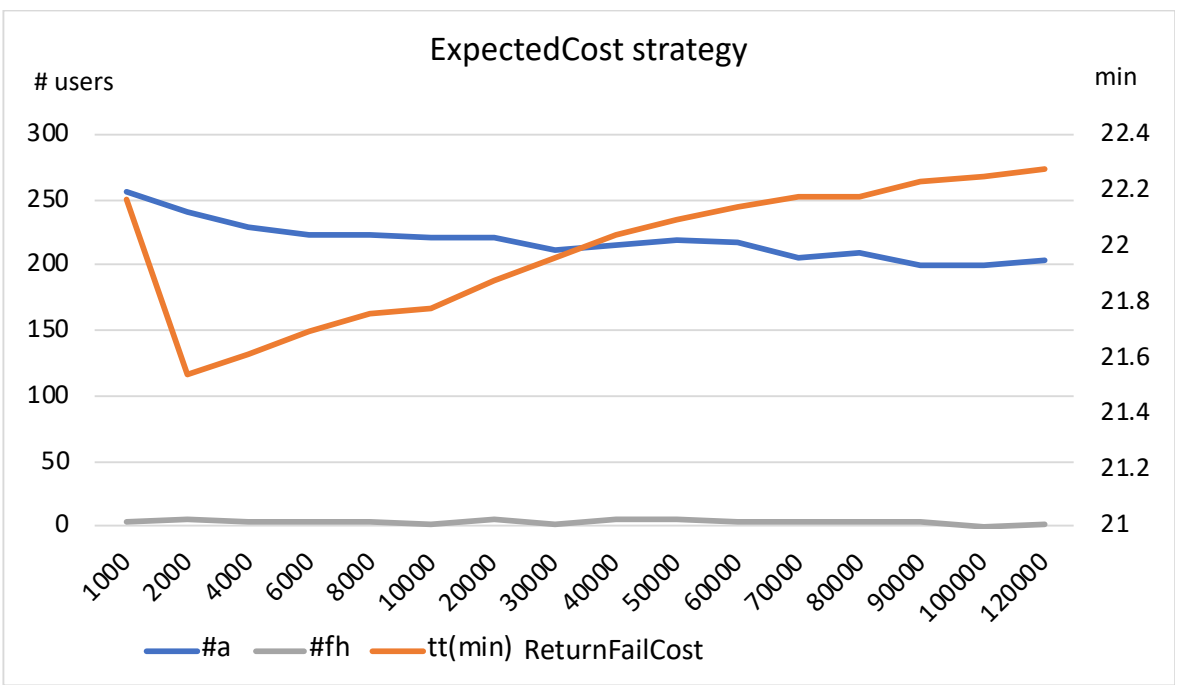

Figure 2. ExpectedCost strategy with fixed RentFailCost $=50,000$ and varying ReturnFailCost.

In Figure 3, we show a fixed value of ReturnFailCost $=2000$ (the value that generally obtains the lowest travel time for different values of RentFailCost), and we increase RentFailCost. As can be seen, higher values of RentFailCost produce lower abandonment rates and fewer failed rental attempts, but lead to increased travel times. It seems that the values remain almost stable at a certain point. 


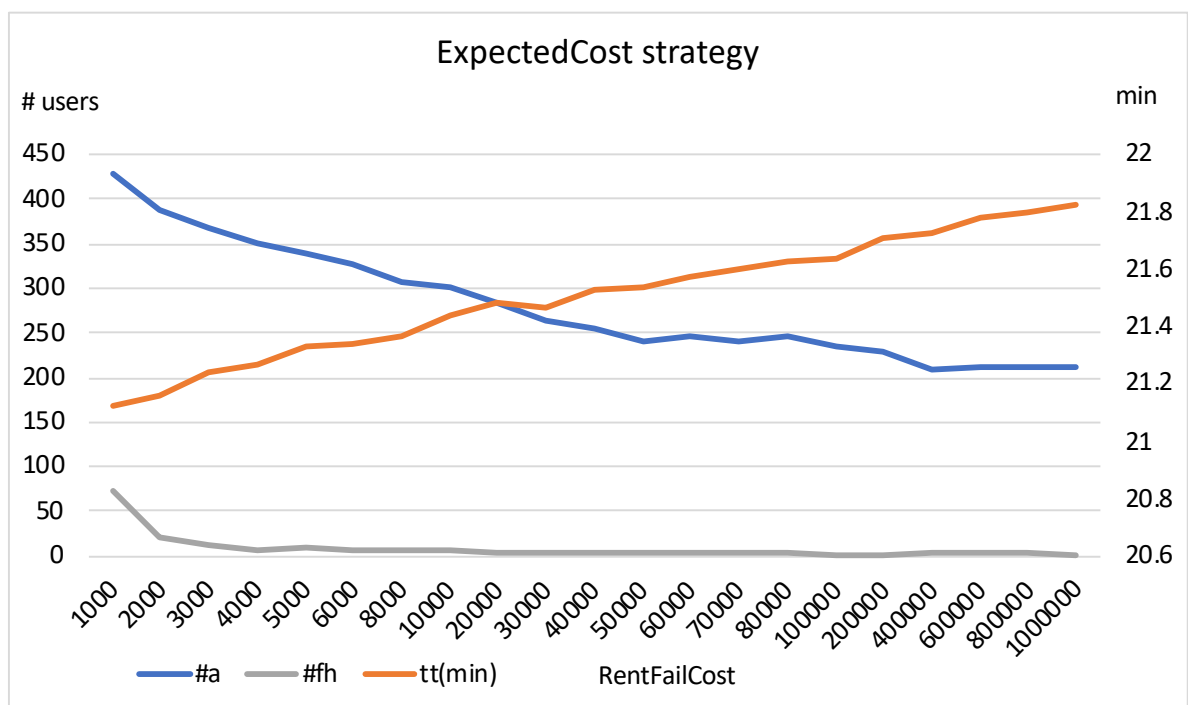

Figure 3. ExpectedCost strategy with variable RentFailCost and fixed ReturnFailCost $=2000$.

If we increase RentFailCost and ReturnFailCost at the same time, as shown in Figure 4, the abandonment rate reduces continuously and considerably, but at the cost of an increase in travel time. The failed rental attempts tend towards 0 .

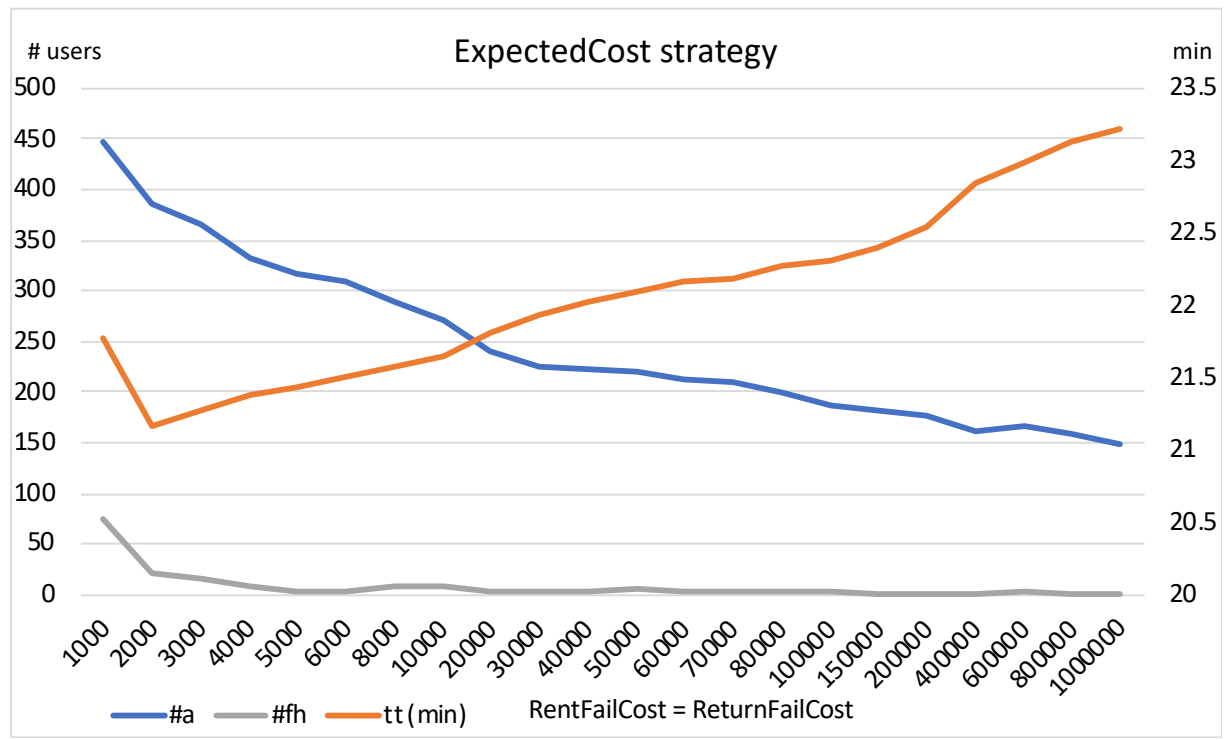

Figure 4. ExpectedCost strategy with increasing equal values of RentFailCost and fixed ReturnFailCost.

Comparing the ExpectedCost strategy with DistanceExpectedResources, the former can be parametrised in order to prioritise abandonment rate or travel time. However, it is interesting to observe that for the same ratio of travel time, both methods obtain almost the same dropout rate, and vice versa. In other words, the rather simple $D E R$ strategy performs as well as the much more sophisticated EC strategy. In our opinion, the explanation of this fact is that the DER strategy not only proposes an appropriate station to rent or return a bike in terms of the utility for an individual user, but also encourages users to take or return bikes at stations with more bikes/slots. In this way, the strategy implicitly improves the unbalancing problem, helping to obtain a better distribution of bikes (and slots) and, thus, to reduce the abandonment rate and the travel time of potential future users. 


\section{Recommendation Based on Local and Global Utility}

As argued previously, the DER strategy with a rather simple calculation model has a quite acceptable performance, because it combines the local utility of a recommendation for a user with a certain improvement of the global situation for the future. In this section, we use this idea to extend the ExpectedCost strategy in the same sense. The idea is to account not only for the cost of a station for a certain user, but also for the changes in the costs to potential future users, if they decide to take (or return) a bike at given station. In particular, taking into account the future demand of bikes or slots at a station, we estimate how taking or returning a bike will increase or decrease the number of expected unsuccessful rental and return attempts of future users at a station within a certain timeframe.

\subsection{Calculating the Future Impact of Rentals and Returns}

To estimate the impact that a bike rental or return has globally on system performance, we analysed how such an event would change the number of expected unsuccessful rental and return attempts of future users-that is, the impact can be measured through the difference in expected unsuccessful events during a given time interval $\left[t_{a}, t_{b}\right]$, if a bike is taken (or returned) at the beginning of this time interval. Moreover, given a station $s_{i}$ and the expected rental rate during the interval $\left[t_{a}, t_{b}\right]$ (obtained by analysing historical demands, for example), the number of expected unsuccessful rental attempts is the number of all rental attempts multiplied by the probability that such attempts fail. We formalize this idea below.

Modelling a station $s_{i}$ as a queue, the average probability of the station being in state 0 - that is, having no available bikes—during a time interval $\left[t_{a}, t_{b}\right]$ with $t_{b}>t_{a}$ is given by:

$$
\bar{\pi}_{0}^{i}\left(t_{a} \rightarrow t_{b}\right)=\frac{1}{t_{b}-t_{a}} \int_{t_{a}}^{t_{b}} \pi_{0}^{i}(w) d w
$$

Based on the system of differential Equations (13)-(15), and given the initial probability distribution $\pi^{i}\left(t_{a}\right)$ and a step-size $h$ with $\left(t_{b}-t_{a}\right)>h>0$, we can use the fourth-order Runge-Kutta method (RK4) to estimate $\bar{\pi}_{0}^{i}\left(t_{a} \rightarrow t_{b}\right)$. Let $\left(\pi_{0}^{i}\left(t_{a}\right), \pi_{0}^{i}\left(t_{a}+h\right), \pi_{0}^{i}\left(t_{a}+2 h\right), \ldots, \pi_{0}^{i}\left(t_{a}+k h\right)\right)$, where $t_{a}+k h=t_{b}$, denote the sequence of iteratively calculated values for $\pi_{0}^{i}(t)$ from $t=t_{a}$ to $t_{b}$ at steps $h$ with RK4. Then, we can use a Riemann sum to approximate $\bar{\pi}_{0}^{i}\left(t_{a} \rightarrow t_{b}\right)$ :

$$
\bar{\pi}_{0}^{i}\left(t_{a} \rightarrow t_{b}\right)=\frac{1}{t_{b}-t_{a}} \sum_{j=0}^{k-1} h \frac{\pi_{0}^{i}\left(t_{a}+j h\right)+\pi_{0}^{i}\left(t_{a}+(j+1) h\right)}{2}
$$

Finally, given the rental appearance rate for a station $s_{i}$ in the interval $\left[t_{a}, t_{b}\right]$, rentDemand $\left(s_{i}, t_{a}, t_{b}\right)$, the expected number of failed rental attempts in the interval is estimated by:

$$
\operatorname{ehf}\left(s_{i}, t_{a}, t_{b}\right)=\bar{\pi}_{0}^{i}\left(t_{a} \rightarrow t_{b}\right) \cdot \operatorname{rentDemand}\left(s_{i}, t_{a}, t_{b}\right)
$$

In a similar way, we can calculate the expected failed return attempts for a station $s_{i}$ in an interval $\left[t_{a}, t_{b}\right]$ :

$$
\operatorname{erf}\left(s_{i}, t_{a}, t_{b}\right)=\bar{\pi}_{c\left(s_{i}\right)}^{i}\left(t_{a} \rightarrow t_{b}\right) \cdot \operatorname{returnDemand}\left(s_{i}, t_{a}, t_{b}\right)
$$

where $c\left(s_{i}\right)$ is the capacity of station $s_{i}$.

If a user $u_{k}$ issues a rental request rent $t_{k}=\left\langle l_{k}, t, m d\right\rangle$, we use ehf and erf to estimate the future impact that a potential rental would have at a station $s_{i}$. The idea is to first calculate the probability distribution of available bikes when the user arrives at the station. Afterwards, we analyse the expected rental and return failures that would arise after the arrival and during a given timeframe $(t f)$ if the user rented out a bike and if they did not do so. The difference between these values can be used as a measure for estimating the future impact of renting a bike at a station. Formally, we first calculate the 
probability distribution of bike availability at the expected arrival time of user $u_{k}: \pi^{i}\left(t_{\exp }\right)$, with $t_{\text {exp }}=t+$ wtime $\left(l_{k}, l\left(s_{i}\right)\right)$, as explained in Section 4. Using $\pi^{i}\left(t_{\text {exp }}\right)$ as the initial distribution, and applying Equations (24)-(26), we can calculate $\operatorname{eh} f\left(s_{i}, t_{\exp }, t_{\exp }+t f\right)$ and $\operatorname{erf}\left(s_{i}, t_{\exp }, t_{\exp }+t f\right)$. Then, we transform from $\pi^{i}\left(t_{\exp }\right)$ to a distribution $\pi^{i, r e n t}\left(t_{\exp }\right)$, where one bike is "taken" away. This means:

$$
\begin{gathered}
\pi_{0}^{i, \text { rent }}\left(t_{\text {exp }}\right)=\pi_{0}^{i}\left(t_{\text {exp }}\right)+\pi_{1}^{i}\left(t_{\text {exp }}\right) \\
\pi_{k}^{i, \text { rent }}\left(t_{\text {exp }}\right)=\pi_{k+1}^{i}\left(t_{\text {exp }}\right), \text { for } c\left(s_{i}\right)>k>0 \\
\pi_{k}^{i, \text { rent }}\left(t_{\text {exp }}\right)=0, \quad \text { for } k=c\left(s_{i}\right)
\end{gathered}
$$

Now, taking $\pi^{i, r e n t}\left(t_{\exp }\right)$ as the initial distribution, we calculate $\operatorname{ehfrent}\left(s_{i}, t_{\exp }, t_{\exp }+t f\right)$ and $\operatorname{erf} f^{r e n t}\left(s_{i}, t_{\text {exp }}, t_{\text {exp }}+t f\right)$. Finally, we can obtain the rental failure impact (ehf Im $\left.{ }^{\text {rent }}\right)$ and return failure impact $\left(\mathrm{erf} \mathrm{Im}^{\text {rent }}\right)$ of the potential rental:

$$
\begin{aligned}
& \operatorname{ehfIm}{ }^{r e n t}\left(u_{k}, s_{i}, t f\right)=\operatorname{ehf} f^{r e n t}\left(s_{i}, t_{\text {exp }}, t_{\text {exp }}+t f\right)-\operatorname{ehf}\left(s_{i}, t_{\text {exp }}, t_{\text {exp }}+t f\right) \\
& \operatorname{erfIm}{ }^{r e n t}\left(u_{k}, s_{i}, t f\right)=\operatorname{erf} f^{r e n t}\left(s_{i}, t_{\text {exp }}, t_{\text {exp }}+t f\right)-\operatorname{erf}\left(s_{i}, t_{\text {exp }}, t_{\text {exp }}+t f\right)
\end{aligned}
$$

It should be noted that $\operatorname{ehf} f^{\text {rent }}\left(s_{i}, t_{\exp }, t_{\exp }+t f\right) \geq \operatorname{eh} f\left(s_{i}, t_{\exp }, t_{\exp }+t f\right)$, because the expected rental failure rate is necessarily higher if a bike is taken from a station; thus, ehf Im ${ }^{\text {rent }}\left(u_{k}, s_{i}, t f\right)$ would be positive. On the other hand, erf $\operatorname{Im}^{\text {rent }}\left(u_{k}, s_{i}, t f\right) \leq 0$, since the number of available slots increases if a bike is taken from the station. Thus, the values of $e h f \operatorname{Im}^{r e n t}\left(u_{k}, s_{i}, t f\right)$ and $\operatorname{erfIm} \operatorname{Im}^{r e n t}\left(u_{k}, s_{i}, t f\right)$ represent the expectation of failed rental and return attempts that are caused by user $u_{k}$ when they take a bike at station $s_{i}$.

In a similar way-but transforming $\pi^{i}\left(t_{\text {exp }}\right)$ to a distribution $\pi^{i \text {,return }}\left(t_{\text {exp }}\right)$, where one bike is "added" - we can calculate the expected failed rental and return attempts that are caused if user $u_{k}$ returns a bike at station $s_{i}$ :

$$
\begin{aligned}
& \operatorname{ehfIm}{ }^{\text {return }}\left(u_{k}, s_{i}, t f\right)=\operatorname{eh} f^{\text {return }}\left(s_{i}, t_{\text {exp }}, t_{\text {exp }}+t f\right)-\operatorname{eh} f\left(s_{i}, t_{\text {exp }}, t_{\text {exp }}+t f\right) \\
& \operatorname{erfIm}{ }^{\text {return }}\left(u_{k}, s_{i}, t f\right)=\operatorname{erf} f^{\text {return }}\left(s_{i}, t_{\text {exp }}, t_{\text {exp }}+t f\right)-\operatorname{erf}\left(s_{i}, t_{\text {exp }}, t_{\text {exp }}+t f\right)
\end{aligned}
$$

\subsection{Recommendation Based on Expected Cost and Future Impact}

We calculate the global cost of renting at a station by combing its local cost with the impact on the overall system in the next given timeframe:

$$
\begin{aligned}
& \text { globalRentCost }\left(s_{i}, \text { rent }_{k}, t f, \text { RentFailCost, FRentFC, FReturnFC }\right)= \\
& \text { localRentCost }\left(s_{i}, \text { rent }_{k}, \text { RentFailCost }+f \cdot \text { bikeProb }_{\left(s_{i}\right.}, \text { rent }_{k}\right) \cdot \\
& \left(\operatorname{ehfIm}{ }^{r e n t}\left(u_{k}, s_{i}, t f\right) \cdot \operatorname{rentCost}\left(s_{i}, l_{k}, t f, F \operatorname{RentFC}\right)+\right. \\
& \left.\operatorname{erfIm}^{\text {rent }}\left(u_{k}, s_{i}, t f\right) \cdot \operatorname{returnCost}\left(s_{i}, l_{k}, t f, \text { FReturnFC }\right)\right)
\end{aligned}
$$

globalReturn Cost $\left(s_{i}\right.$, return $_{k}, t f$, ReturnFailCost, FRentFC, FReturnFC $)=$ localReturn Cost $\left(s_{i}\right.$, return $_{k}$, ReturnFailCost $)+$

$f \cdot \operatorname{slotProb}\left(s_{i}, \operatorname{return}_{k}\right) \cdot\left(\operatorname{ehfIm} \operatorname{Im}^{\text {return }}\left(u_{k}, s_{i}, t f\right) \cdot \operatorname{rentCost}\left(s_{i}, l_{k}, t f, F \operatorname{RentFC}\right)+\operatorname{erfIm}{ }^{\text {return }}\left(u_{k}, s_{i}, t f\right) \cdot \operatorname{return} \operatorname{Cost}\left(s_{i}, l_{k}, t f, F \operatorname{ReturnFC}\right)\right)$

$f$ is a parameter that assigns more or less importance to the local cost versus the global impact. rentCost and returnCost represent the cost associated with the number of extra future rental and return failures, respectively. This cost should depend on whether a potential user who arrives at a station and fails to rent or to return a bike has some alternative to rent or return a bike at a station nearby. With this idea, we define:

$$
\begin{gathered}
\operatorname{rentCost}\left(s_{i}, l_{k}, t f, F \operatorname{RentFC}\right)= \\
\min _{s_{j} \in S \wedge \operatorname{wist}\left(s_{i}, s_{j}\right) \leq M D} \text { localRentCost }\left(s_{j},<l\left(s_{i}\right), t^{\prime}, M D,>, F \operatorname{RentFC}\right) \\
F \operatorname{RentFC}, \text { if } \neg \exists s_{j} \in S: \operatorname{wdist}\left(s_{i}, s_{j}\right) \leq M D
\end{gathered}
$$




$$
\begin{aligned}
& \text { returnCost }\left(s_{i}, l_{k}, t f, \text { FReturnFC }\right)= \\
& \min _{s_{j} \in S \wedge \operatorname{dist}\left(s_{i}, s_{j}\right) \leq M D} \text { localReturnCost }\left(s_{j},<l\left(s_{i}\right), t^{\prime \prime}, M D>, \text { FReturnFC }\right) \\
& \text { FReturnFC, if } \neg \exists s_{j} \in S: \operatorname{bdist}\left(s_{i}, s_{j}\right) \leq M D
\end{aligned}
$$

where $t^{\prime}=t+$ wtime $\left(l_{k}, l\left(s_{i}\right)\right)+t f / 2$ and $t^{\prime \prime}=t+b$ time $\left(l_{k}, l\left(s_{i}\right)\right)+t f / 2$. This means that rentCost is the lowest local cost of stations in the vicinity of station $s_{i}$ (within a distance of $M D$ meters), and taking the parameter FRentFC as the penalization cost. In the worst case-e.g., when no station exists within a distance of $M D$ meters from $s_{i}$ - the value of rentCost will be FRentFC. In the same way, returnCost will be at most FReturnFC. Note that the local costs for users who would arrive at station $s_{i}$ are appproximated by considering they arrive at mid timeframe after the arrival of user $u_{k}$.

Based on the global costs, we define the recommendation strategy ExpectedCostFutureImpact:

$$
\operatorname{RentStation}\left(\text { rent }_{k}\right)=\left(s_{i}\right)_{i=1}^{m}
$$

where:

$$
\begin{aligned}
& \forall s_{i}: \text { wdist }\left(l_{k}, l\left(s_{i}\right)\right) \leq m d \wedge \\
& \forall s_{i}, s_{j}, i<j: \\
& \text { globalRentCost }\left(s_{i}, \text { rent }_{k}, t f, \text { RentFailCost, FRentFC, FReturnFC }\right) \leq \\
& \text { globalRentCost }\left(s_{j}, \text { rent }_{k}, t f, \text { RentFailCost, FRentFC, FReturnFC }\right) \\
& \left.\qquad \operatorname{ReturnStation}_{\left(\text {return }_{k}\right)}\right)=\left(s_{i}\right)_{i=1}^{m},
\end{aligned}
$$

where:

$$
\begin{aligned}
& \forall s_{i}, s_{j}, i<j: \\
& \text { globalReturn Cost }\left(s_{i}, \text { return }_{k}, t f, \text { ReturnFailCost, FRentFC, FReturnFC }\right) \leq \\
& \text { globalReturn Cost }\left(s_{j}, \text { return }_{k}, t f, \text { ReturnFailCost, FRentFC, FReturnFC }\right)
\end{aligned}
$$

In summary, the parameters of this strategy are:

- $\quad m d$ - the maximum distance to rental stations;

- RentFailCost and ReturnFail Cost-the penalization costs applied if a user is unsuccessful when trying to rent or return a bike;

- $\quad t f$-the timeframe for predicting the future impact of a rental or return action;

- $\quad F R e n t F C$ and FReturnFC - the penalization costs applied when estimating the costs of local alternative stations for users who cannot find a bike or slot at station $s_{i}$ in the future;

- $M D$ - the maximum distance to consider alternative stations for users who cannot find a bike or slot at station $s_{i}$;

- $\quad f$-the factor applied to the global impact on the cost estimation.

\section{Evaluation of ExpectedCostFutureImpact Recommendation}

We used the same settings as in Section 5 to evaluate the ExpectedCostFutureImpact strategy. In the experiments, we fixed the following parameters: the values of $m d=600 \mathrm{~m}$, as a reasonable distance that a user might be willing to walk to get a bike, and $M D=500 \mathrm{~m}$.

In the first set of experiments, we analysed this approach with different penalization costs, where the timeframe $t f$ was set to $1 \mathrm{~h}$ and the factor $f=1$. Some results are presented in Table 4. 
Table 4. Experimental results for Madrid. Bold numbers indicate the best obtained result for each metric.

\begin{tabular}{cccccc}
\hline $\begin{array}{c}\text { RentFailCost/ReturnFailCost } \\
\text { /FutRentFailCost } \\
\text { /FutReturnFailCost }\end{array}$ & \#a & $\# \mathrm{fh}$ & $\# \mathrm{fr}$ & $\mathbf{t t}$ (min) & AET (min) \\
\hline $1000 / 2000 / 1000 / 2000$ & 193 & 71 & 87 & 21.04 & 223.3 \\
\hline $3000 / \mathbf{2 5 0 0 / 3 0 0 0 / 1 0 0 0}$ & 15 & 2 & 20 & 21.63 & 128.0 \\
\hline $3000 / 2000 / 3000 / 2000$ & 29 & 3 & 83 & 21.70 & 132.5 \\
\hline $70,000 / 2000 / 70,000 / 2000$ & 27 & 0 & 734 & 29.25 & 24.22 \\
\hline $1,000,000 / 1,000,000 / 1,000,000 / 1,000,000$ & 22 & 0 & 209 & 40.61 & 18.13 \\
\hline $100,000 / 100,000 / 100,000 / 100,000$ & 10 & 0 & 162 & 28.99 & 11.67 \\
\hline $50,000 / 50,000 / 50,000 / 50,000$ & 14 & 0 & 124 & 27.08 & 13.11 \\
\hline $10,000 / 10,000 / 10,000 / 10,000$ & 20 & 1 & 15 & 23.27 & 40.79 \\
\hline $5000 / 5000 / 5000 / 5000$ & 22 & 1 & 1 & 22.37 & 83.30 \\
\hline $3000 / 3000 / 3000 / 3000$ & 35 & 1 & 6 & 21.80 & 127.9 \\
\hline
\end{tabular}

Analysing the results presented in the table, we can observe that, in general, higher penalization costs lead to lower dropout rates but longer travel times, whereas lower costs have the opposite effect: more abandonments and shorter travel times. A good combination of both metrics can be obtained with values close to the following: RentFail Cost $=3000$, ReturnFailCost $=2500$, FutRentFailCost $=3000$, and FutReturnFailCost $=1000$. In particular, the abandonment rate can be reduced considerably if a recommendation considers not only the local cost for a user (to rent or return a bike), but also the impact on potential users in the future. For example, the strategy with the penalization schema $3000 / 2500 / 3000 / 1000$, as compared to $E C(70,000 / 2000)$, reduces the number of abandonments from 240 to 15 by maintaining the same travel time (21.6 min). In Section 5, the EC (70,000/2000) strategy showed a good behaviour in terms of abandonment rate and low travel time.

Using the penalization cost pattern 3000/2500/3000/1000, and fixing the factor $f=1$, in Figure 5 we analyse the influence of the prediction timeframe in the results.

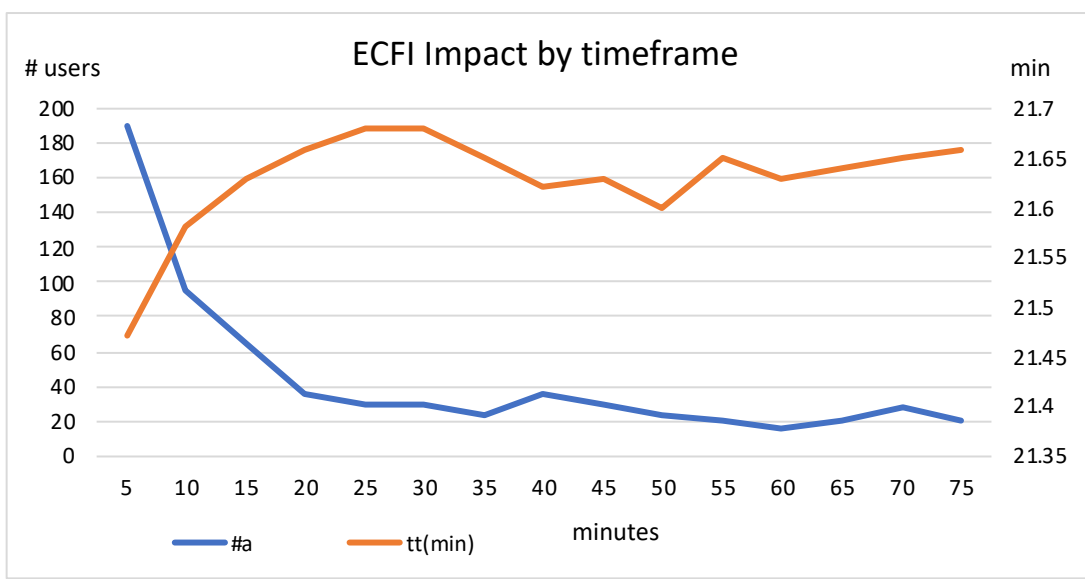

Figure 5. Impact of different prediction timeframes on the behaviour of ExpectedCostFutureImpact (with RentFailCost $=3000$, ReturnFailCost $=2500$, FutRentFailCost $=3000$, FutReturnFailCost $=1000$, and $f=1$ ).

Figure 5 indicates that, as expected, short prediction timeframes present higher abandonment rates but faster travel times. It is more likely that future users will not find an available bike or slot, but users are sent to "closer" stations since the considered future impact will be lower. At some point, the values for dropout rate and travel time tend to 
stabilize, and even increase slightly. Good results are obtained with a prediction timeframe of $\sim 20 \mathrm{~min}$.

The best way to prioritise between average travel time and abandonment rate is via the factor $f$. In Figure 6, we show different results obtained when varying $f$. Low values for $f$ decrease travel time, but lead to more abandonments. A good compromise is obtained with $f$ around 1 .

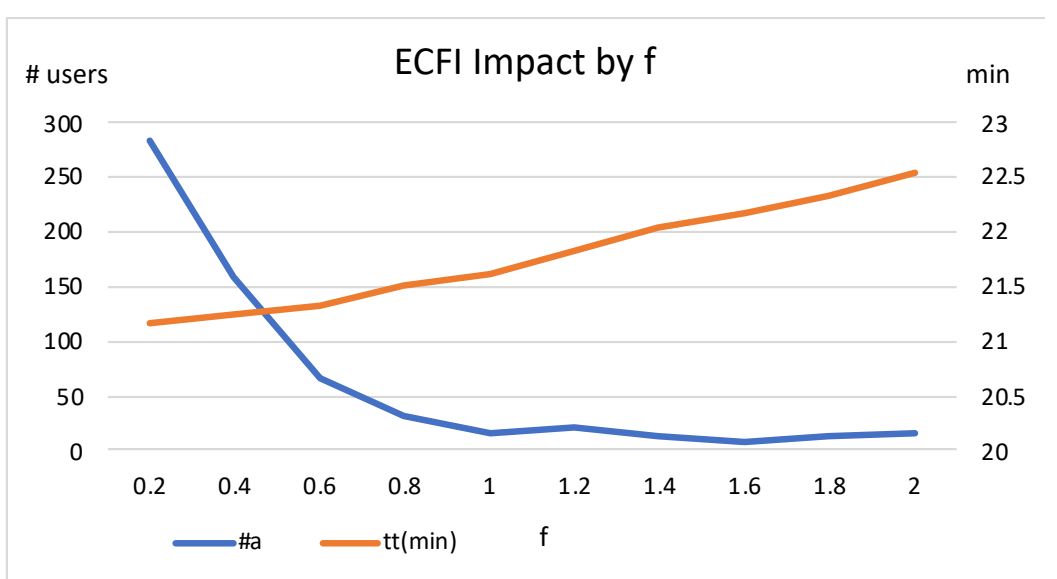

Figure 6. Impact of different factors $f$ with ExpectedCostFutureImpact (with RentFailCost $=3000$, ReturnFailCost $=2500$, FutRentFailCost $=3000$, FutReturnFailCost $=1000$, and $t f=60 \mathrm{~min}$ ).

As we mentioned in Section 5, the experiments in this paper are based on real data, but we reduced the station capacities and the overall number of bikes in the system to approximately half of their original values. The reason for this was that such a situation is more challenging and, thus, the differences in the recommendation strategies are more appreciable. In Table 5, however, we present and compare the standard strategies with the best variations of the ExpectedCost and the ExpectedCostFutureImpact strategies, in a scenario with the original (real) station capacities and bike numbers. As expected, all strategies significantly improved their performance with real station capacities. As shown, the novel strategy ExpectedCostFutureImpact proposed in this paper also performs better than the other strategies in this situation, in terms of both abandonment rate and travel time.

Table 5. Comparison of strategies with real station capacities and number of bikes.

\begin{tabular}{cccccc}
\hline Strategy & \#a & \#fh & \#fr & tt (min) & $\begin{array}{c}\text { AET } \\
(\mathbf{m i n})\end{array}$ \\
\hline OPTIMUM & 0 & 0 & 0 & 19.21 \\
\hline SD & 262 & 697 & 1613 & 20.22 & 143.75 \\
\hline ISD & 115 & 90 & 437 & 19.85 & 145.75 \\
\hline AR & 24 & 0 & 17 & 27.99 & 3.98 \\
\hline DR & 38 & 7 & 18 & 20.01 & 37.55 \\
\hline DER $(3000 / 2000)$ & 18 & 0 & 0 & 20.01 & 32.87 \\
\hline $\begin{array}{c}\text { ECFI }(\text { RentFailCost }=3000, \text { ReturnFailCost }=2500, \\
\text { 1000, } t f=60 \text { min and } f=1\end{array}$ & 41 & 0 & 1 & 19.52 & 87.89 \\
\hline
\end{tabular}

\section{Conclusions}

Bike-sharing systems are becoming an integral part of intelligent transportation infrastructure in smart cities. Station-based BSSs have the advantage of being more resilient with regards to misuse and vandalism, and also account for seamless charging if the BSS fleet contains electric bicycles. A typical problem in station-based BSSs is the possibility that 
some stations may run out of available resources due to high and unbalanced demands at peak times. However, if no bikes are available near their location, or if finding an available parking slot at the destination is difficult, users may drop out of the BSS and use other, less eco-friendly means of transportation.

In this paper, we addressed the balancing problem in BSSs by developing recommendation strategies that help users to select a station to rent or return a bike, considering the distance/time to that station as well as the likelihood that they will find a bike/slot when they actually arrive at the station.

Our contribution is twofold: In the first part of the paper, we presented and analysed station selection (or recommendation) strategies that are user-centred-that is, they try to find the best station considering only the utility or expected cost of a specific user. We presented the DistanceExpectedResources strategy, which assumes that recent recommendations are being followed by users and, thus, can better estimate the resources that are expected to be available at the moment when a user actually arrives at a certain station. We also presented the ExpectedCost strategy, which minimizes a user's cost, combining the distance from their origin (or destination) to the location of candidate stations, and the probability of finding an available bike (or empty slot) when they arrive. This strategy models stations as queues and uses demand data to estimate the probability of finding available bikes or slots. We compared the performance of the presented strategies through simulation experiments with real data from the BiciMAD BSS in Madrid. Both methods outperform baseline station selection strategies such as "going to the closest" or "going to the closest station with available bikes/slots", in terms of both (1) the number of users who abandon the system without renting a bike, and (2) the total time in the system.

In the second part of the paper, we proposed a station recommendation strategy that seeks an equilibrium between local (user-centred) and global utility. With regard to the latter, recommendations prioritise stations that are good for a particular user, but also imply some positive impact on the distribution of bikes and slots in the overall system, and for potential future users. In particular, we defined the ExpectedCostFutureImpact strategy, which extends the ExpectedCost approach by also analysing the impact that choosing a particular station will have on future rentals and returns. In the simulation experiments, this solution outperformed all other strategies, with respect to both the number of abandonments and total travel time.

In future works, we aim to look into explicit (e.g., monetary) incentive mechanisms so as to persuade individually rational users to follow our recommendations while maintaining their trust in the system. These incentives are likely to be proportional in some way to the globalRentCost. Another interesting line for future research in this context consists of learning, from experience, the likelihood that specific (types of) users will follow the recommendations given. This would allow a more fine-grained adjustment of our recommendation model based on user profiles, and could also be used to implement specific user-centred incentives. Finally, as we mentioned in the introduction, BSSs could be conceived as part of a sophisticated multimodal intelligent transportation solution. The synergic effects within such a system of systems open up a whole range of new opportunities, especially with regards to better availability of green transportation services and a reduced number of dropouts.

Author Contributions: Conceptualization, H.B., A.F. and S.O.; methodology, H.B., A.F. and S.O.; software, H.B.; validation, H.B., A.F. and S.O.; formal analysis, H.B., A.F. and S.O.; investigation, H.B., A.F. and S.O.; writing—original draft preparation, H.B., A.F. and S.O.; writing-review and editing, H.B., A.F. and S.O.; visualization, H.B., A.F. and S.O.; funding acquisition, H.B., A.F. and S.O. All authors have read and agreed to the published version of the manuscript.

Funding: This work has been partially supported by the Spanish Ministry of Science, Innovation, and Universities, co-funded by EU FEDER Funds, through grant RTI2018-095390-B-C33 (MCIU/AEI/ FEDER, UE).

Institutional Review Board Statement: Not applicable. 
Informed Consent Statement: Not applicable.

Data Availability Statement: Not applicable.

Conflicts of Interest: The authors declare no conflict of interest.

\section{References}

1. Soriguera, F.; Meroño, E.J. A continuous approximation model for the optimal design of public bike-sharing systems. Sustain. Cities Soc. 2020, 52, 101826. [CrossRef]

2. Ţuţu, I.; Chiriţă, C.E.; Lopes, A.; Fiadeiro, J.L. Logical Support for Bike-Sharing System Design. In Lecture Notes in Computer Science (Including Subseries Lecture Notes in Artificial Intelligence and Lecture Notes in Bioinformatics); Springer: Cham, Switzerland, 2019; Volume 11865; pp. 152-171. [CrossRef]

3. Diez, C.; Palanca, J.; Sanchez-Anguix, V.; Heras, S.; Giret, A.; Julián, V. Towards a Persuasive Recommender for Bike Sharing Systems: A Defeasible Argumentation Approach. Energies 2019, 12, 662. [CrossRef]

4. Binetti, M.; Caggiani, L.; Camporeale, R.; Ottomanelli, M. A Sustainable Crowdsourced Delivery System to Foster Free-Floating Bike-Sharing. Sustainability 2019, 11, 2772. [CrossRef]

5. Aguiari, D.; Delnevo, G.; Monti, L.; Ghini, V.; Mirri, S.; Salomoni, P.; Pau, G.; Im, M.; Tse, R.; Ekpanyapong, M.; et al. Canarin II: Designing a smart e-bike eco-system. In Proceedings of the 15th IEEE Annual Consumer Communications \& Networking Conference (CCNC), Las Vegas, NV, USA, 12-15 January 2018; pp. 1-6. [CrossRef]

6. Voinea, S.C.; Bujari, A.; Palazzi, C.E. Air Quality Control through Bike Sharing Fleets. In Proceedings of the IEEE Symposium on Computers and Communications (ISCC), Rennes, France, 7-10 July 2020; pp. 1-4. [CrossRef]

7. Al-Rahamneh, A.; Astrain, J.J.; Villadangos, J.; Klaina, H.; Guembe, I.P.; Lopez-Iturri, P.; Falcone, F. Enabling Customizable Services for Multimodal Smart Mobility with City-Platforms. IEEE Access 2021, 9, 41628-41646. [CrossRef]

8. Bin Hariz, M.; Said, D.; Mouftah, H.T. Game Theoretic Approach for a Multi-Mode Transportation in Smart Cities. In Proceedings of the 2020 International Symposium on Networks, Computers and Communications (ISNCC), Montreal, QC, Canada, 20-22 October 2020; pp. 1-6. [CrossRef]

9. Sanchez-Iborra, R.; Bernal-Escobedo, L.; Santa, J. Eco-Efficient Mobility in Smart City Scenarios. Sustainability 2020, $12,8443$. [CrossRef]

10. Cepeliauskaite, G.; Keppner, B.; Simkute, Z.; Stasiskiene, Z.; Leuser, L.; Kalnina, I.; Kotovica, N.; Andinš, J.; Muiste, M. SmartMobility Services for Climate Mitigation in Urban Areas: Case Studies of Baltic Countries and Germany. Sustainability 2021, 13, 4127. [CrossRef]

11. Aziz, H.A.; Park, B.H.; Morton, A.; Stewart, R.N.; Hilliard, M.; Maness, M. A high resolution agent-based model to support walk-bicycle infrastructure investment decisions: A case study with New York City. Transp. Res. Part C Emerg. Technol. 2018, 86, 280-299. [CrossRef]

12. Lin, Y.-K.; Liang, F. Simulation for Balancing Bike-Sharing Systems. Int. J. Model. Optim. 2017, 7, $24-27$.

13. Saltzman, R.M.; Bradford, R.M. Simulating a More Efficient Bike Sharing System. J. Supply Chain Oper. Manag. $2016,14,36-47$.

14. Cintrano, C.; Chicano, F.; Alba, E. Using metaheuristics for the location of bicycle stations. Expert Syst. Appl. 2020, 161, 113684. [CrossRef]

15. Reck, D.J.; Haitao, H.; Guidon, S.; Axhausen, K.W. Explaining shared micromobility usage, competition and mode choice by modelling empirical data from Zurich, Switzerland. Transp. Res. Part C Emerg. Technol. 2021, 124, 102947. [CrossRef]

16. Forma, I.A.; Raviv, T.; Tzur, M. A 3-step math heuristic for the static repositioning problem in bike-sharing systems. Transp. Res. Part B Methodol. 2015, 71, 230-247. [CrossRef]

17. Jian, N.; Freund, D.; Wiberg, H.M.; Henderson, S.G. Simulation optimization for a large-scale bike-sharing system. In Proceedings of the Winter Simulation Conference (WSC), Washington, DC, USA, 11-14 December 2016; pp. 602-613. [CrossRef]

18. Bulhões, T.; Subramanian, A.; Erdoğan, G.; Laporte, G. The static bike relocation problem with multiple vehicles and visits. Eur. J. Oper. Res. 2018, 264, 508-523. [CrossRef]

19. Chemla, D.; Meunier, F.; Pradeau, T.; Calvo, R.W.; Yahiaoui, H. Self-Service Bike Sharing Systems: Simulation, Repositioning, Pricing. Available online: https:/ / hal.archives-ouvertes.fr/hal-00824078 (accessed on 15 October 2021).

20. O'Mahony, E.; Shmoys, D.B. Data analysis and optimization for (citi)bike sharing. In Proceedings of the Twenty-Ninth AAAI Conference on Artificial Intelligence (AAAI'15), Austin, TX, USA, 25-30 January 2015; pp. 687-694.

21. Schuijbroek, J.; Hampshire, R.; van Hoeve, W.-J. Inventory rebalancing and vehicle routing in bike sharing systems. Eur. J. Oper. Res. 2017, 257, 992-1004. [CrossRef]

22. Affonso, R.C.; Couffin, F.; LeClaire, P. Modelling of User Behaviour for Static Rebalancing of Bike Sharing System: Transfer of Demand from Bike-Shortage Stations to Neighbouring Stations. J. Adv. Transp. 2021, 2021, 8825521. [CrossRef]

23. Pfrommer, J.; Warrington, J.; Schildbach, G.; Morari, M. Dynamic Vehicle Redistribution and Online Price Incentives in Shared Mobility Systems. IEEE Trans. Intell. Transp. Syst. 2014, 15, 1567-1578. [CrossRef]

24. Haider, Z.; Nikolaev, A.; Kang, J.E.; Kwon, C. Inventory rebalancing through pricing in public bike sharing systems. Eur. J. Oper. Res. 2018, 270, 103-117. [CrossRef] 
25. Singla, A.; Santoni, M.; Bartók, G.; Mukerji, P.; Meenen, M.; Krause, A. Incentivizing users for balancing bike sharing systems. In Proceedings of the Twenty-Ninth AAAI Conference on Artificial Intelligence (AAAI'15), Austin, TX, USA, 25-30 January 2015; pp. 723-729.

26. Reiss, S.; Bogenberger, K. Optimal bike fleet management by smart relocation methods: Combining an operator-based with an user-based relocation strategy. In Proceedings of the IEEE 19th International Conference on Intelligent Transportation Systems (ITSC), Rio de Janeiro, Brazil, 1-4 November 2016; pp. 2613-2618. [CrossRef]

27. Wang, I.-L.; Hou, C.-T. A Crowdsourced Dynamic Repositioning Strategy for Public Bike Sharing Systems. Numerical Algebra, Control and Optimization. Available online: https://www.researchgate.net/publication/347915948_A_crowdsourced_dynamic_ repositioning_strategy_for_public_bike_sharing_systems (accessed on 15 October 2021).

28. Chung, H.; Freund, D.; Shmoys, D.B. Bike angels: An analysis of citi bike's incentive program. In Proceedings of the 1st ACM SIGCAS Conference on Computing and Sustainable Societies, New York, NY, USA, 20-22 June 2018; Article 5.

29. O'Mahony, E.D. Smarter Tools for (Citi)Bike Sharing. Ph.D. Thesis, Cornell University, Ithaca, NY, USA, August 2015.

30. Merugu, D.; Prabhakar, B.S.; Rama, N.S. An incentive mechanism for decongesting the roads: A pilot program in bangalore. In Proceedings of the ACM Workshop on the Economics of Networked Systems, Standford, CA, USA, 7 July 2009.

31. Fricker, C.; Gast, N. Incentives and redistribution in homogeneous bike-sharing systems with stations of finite capacity. EURO J. Transp. Logist. 2016, 5, 261-291. [CrossRef]

32. Chiariotti, F.; Pielli, C.; Zanella, A.; Zorzi, M. A Bike-sharing Optimization Framework Combining Dynamic Rebalancing and User Incentives. ACM Trans. Auton. Adapt. Syst. 2020, 14, 1-30. [CrossRef]

33. Li, L.; Shan, M. Bidirectional Incentive Model for Bicycle Redistribution of a Bicycle Sharing System during Rush Hour. Sustainability 2016, 8, 1299. [CrossRef]

34. Yoon, J.W.; Pinelli, F.; Calabrese, F. Cityride: A predictive bike sharing journey advisor. In Proceedings of the IEEE 13th International Conference on Mobile Data Management (MDM), Bengaluru, India, 23-26 July 2012; pp. 306-311.

35. Waserhole, A.; Jost, V. Pricing in vehicle sharing systems: Optimization in queuing networks with product forms. EURO J. Transp. Logist. 2016, 5, 293-320. [CrossRef]

36. López Santiago, A.; Iglesias, C.A.; Carrera, A. Improving Sustainable Mobility with a Variable Incentive Model for Bike-Sharing Systems Based on Agent-Based Social Simulation. In Advances in Practical Applications of Agents, Multi-Agent Systems, and Trustworthiness; The PAAMS Collection; Springer: Cham, Switzerland, 2020; pp. 158-170. [CrossRef]

37. Fernández, A.; Billhardt, H.; Ossowski, S.; Sánchez, O. Bike3S: A tool for bike sharing systems simulation. J. Simul. 2020, 14, 278-294. [CrossRef]

38. Levine, R.V.; Norenzayan, A. The Pace of Life in 31 Countries. J. Cross Cult. Psychol. 1999, 30, 178-205. [CrossRef]

39. Mohler, B.J.; Thompson, W.B.; Creem-Regehr, S.H.; Pick, H.L.; Warren, W.H. Visual flow influences gait transition speed and preferred walking speed. Exp. Brain Res. 2007, 181, 221-228. [CrossRef] [PubMed] 